\title{
M-CFIS-R: Mamdani Complex Fuzzy Inference System with Rule Reduction Using Complex Fuzzy Measures in Granular Computing
}

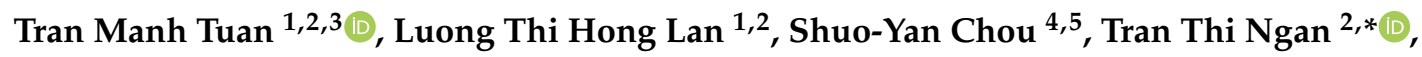 \\ Le Hoang Son ${ }^{6}$, Nguyen Long Giang ${ }^{3}$ and Mumtaz Ali ${ }^{7}$ \\ 1 Vietnam Academy of Science and Technology, Graduate University of Science and Technology, \\ Hanoi 010000, Vietnam; tmtuan@tlu.edu.vn (T.M.T.); lanlhbk@tlu.edu.vn (L.T.H.L.) \\ 2 Faculty of Computer Science and Engineering, Thuyloi University, 175 Tay Son, Dong Da, \\ Hanoi 010000, Vietnam \\ 3 Institute of Information Technology, Vietnam Academy of Science and Technology, Hanoi 010000, Vietnam; \\ nlgiang@ioit.ac.vn \\ 4 Department of Industrial Management, National Taiwan University of Science and Technology, No. 43, \\ Section 4, Keelung Road, Taipei 10607, Taiwan; sychou@mail.ntust.edu.tw \\ 5 Taiwan Building Technology Center, National Taiwan University of Science and Technology, No. 43, \\ Section 4, Keelung Road, Taipei 10607, Taiwan \\ 6 VNU Information Technology Institute, Vietnam National University, Hanoi 010000, Vietnam; \\ sonlh@vnu.edu.vn \\ 7 School of Information Technology, Deakin University, 221 Burwood Highway, \\ Burwood Victoria 3125, Australia; mumtaz.ali@deakin.edu.au \\ * Correspondence: ngantt@tlu.edu.vn; Tel.: +(84)-989-040454
}

Received: 10 April 2020; Accepted: 22 April 2020; Published: 3 May 2020

\begin{abstract}
Complex fuzzy theory has strong practical background in many important applications, especially in decision-making support systems. Recently, the Mamdani Complex Fuzzy Inference System (M-CFIS) has been introduced as an effective tool for handling events that are not restricted to only values of a given time point but also include all values within certain time intervals (i.e., the phase term). In such decision-making problems, the complex fuzzy theory allows us to observe both the amplitude and phase values of an event, thus resulting in better performance. However, one of the limitations of the existing M-CFIS is the rule base that may be redundant to a specific dataset. In order to handle the problem, we propose a new Mamdani Complex Fuzzy Inference System with Rule Reduction Using Complex Fuzzy Measures in Granular Computing called M-CFIS-R. Several fuzzy similarity measures such as Complex Fuzzy Cosine Similarity Measure (CFCSM), Complex Fuzzy Dice Similarity Measure (CFDSM), and Complex Fuzzy Jaccard Similarity Measure (CFJSM) together with their weighted versions are proposed. Those measures are integrated into the M-CFIS-R system by the idea of granular computing such that only important and dominant rules are being kept in the system. The difference and advantage of M-CFIS-R against M-CFIS is the usage of the training process in which the rule base is repeatedly changed toward the original base set until the performance is better. By doing so, the new rule base in M-CFIS-R would improve the performance of the whole system. Experiments on various decision-making datasets demonstrate that the proposed M-CFIS-R performs better than M-CFIS.
\end{abstract}

Keywords: complex fuzzy set; similarity measure; complex fuzzy measure; Mamdani Complex Fuzzy Inference System (M-CFIS); rule reduction; granular computing 


\section{Introduction}

Zadeh [1] proposed fuzzy set (FS) as an approach for representing and processing vagueness found abundantly in the real world. Fuzzy inference systems (FIS) are used to generate fuzzy rule sets, which are applied in solving problems in various applications such as detection [2,3], prediction $[4,5]$, classification [6-8], and other tasks [9-14]. A Complex Fuzzy Set (CFS) [15] is an extension of the fuzzy set, where the membership function consists of both the amplitude term and the phase term. Building upon this, Ramot et al. [16] proposed a novel framework for logical reasoning, termed Complex Fuzzy Logic (CFL), using the CFS theory. Although the CFS and the extensions of the CFS were not applied directly in applications, CFSs were considered as a basic concept to make intelligent systems capable of handling different issues [17-24].

Recently, the Mamdani Complex Fuzzy Inference System (M-CFIS) was proposed in [23]. Some other FISs in the CFS were also developed such as the Adaptive Neuro-complex Fuzzy Inferential System (ANCFIS) with higher-order TSK models [25], Randomized Adaptive Neuro-complex Fuzzy Inference System (RANCFIS) [26], and Fast Adaptive Neuro-complex Fuzzy Inference System (FANCFIS) [27]. However, a potential impairment of the existing M-CFIS is that the rule base may be redundant to a specific dataset. In order to remedy the problem, fuzzy similarity measures should be utilized.

The measures of the CFS were presented in [28], including complex fuzzy distances and distance measures between two CFSs. The distance measures of the CFS were introduced in $[29,30]$. Setnes et al. [31] proposed a similarity measure in fuzzy rule base to evaluate the equality between two fuzzy sets and to simplify the rule base. Similarity measures in complex neutrosophic sets were presented in [32], including Cosine, Dice, and Jaccard similarity measures. The candidates of multi-attribute decision-making were assessed by these measures. Apart from that, the measures based on FS, CFS, or FIS were also used to calculate weights of criteria in a decision-making system [33]. In the CFS, the complex fuzzy measure was defined as the cardinality of fuzzy rule set [29]. The complex fuzzy measures (t-norm and t-conorm) in Mamdani CFIS (M-CFIS) were introduced in [23], where the obtained rule set in M-CFIS directly affects to the results of decision-making. In most cases, there is redundancy in the rule base obtained from M-CFIS.

This paper proposes a new Mamdani Complex Fuzzy Inference System with Rule Reduction Using Complex Fuzzy Measures in Granular Computing called M-CFIS-R. Several fuzzy similarity measures, including Complex Fuzzy Cosine Similarity Measure (CFCSM), Complex Fuzzy Dice Similarity Measure (CFDSM), and Complex Fuzzy Jaccard Similarity Measure (CFJSM) together with their weighted versions are proposed. Those measures are integrated into the M-CFIS-R system by the idea of Granular Computing where only important and dominant rules will be kept in the system. These complex fuzzy measures are used to evaluate the similarity among complex fuzzy rules in the rule set of M-CFIS. Based on the values of these measures, the rules with high similarity will be reduced to guarantee high performance. The advantage of M-CFIS-R over M-CFIS is the usage of the training process in which the rule base is repeatedly changed toward the original base set until the performance is better. By doing so, the new rule base in M-CFIS-R would improve the performance of the whole system. The performance of proposed method is experimentally validated on various decision-making datasets.

\section{Related Works}

\subsection{Complex Fuzzy Measures}

Most research on complex fuzzy measure has mainly focused on certain aspects $[34,35]$ such as the fuzzy measure and classical theory of complex fuzzy numbers, similarity, and distance with the CFS $[29,30]$. 
In a fuzzy rule base, the concept of similarity measure was mentioned by Setnes et al. [31]. Based on this, the similarity of two fuzzy sets was defined. Then, similar fuzzy sets were removed and the common presentations were kept in the rule base. Ma and $\mathrm{Li} \mathrm{[36]} \mathrm{extended} \mathrm{the} \mathrm{classical} \mathrm{measure} \mathrm{to}$ fuzzy complex number valued measure and defined some important properties on complex fuzzy set valued complex fuzzy measures. These properties generalized the corresponding results in measure theory and on the related integral theory. In another study [37], Ma et al. proposed a new concept of complex fuzzy measure, which is distinguished between the real and imaginary. Based on the complex fuzzy measure, $\mathrm{Ma}$ and $\mathrm{Li}$ [38] focused on the convergence problem of the complex fuzzy integral. Alkouri and Salleh [28] introduced the definitions of linguistic variables and linguistic hedges on the CFS. In this research, they also presented several distance measures in the CFS, which might be used as a suggestion in decision-making, prediction, and pattern recognition to find optimal solutions. Other measures and operations on other types of the complex fuzzy set were also presented in [23,29].

The information measure in Complex Intuitionistic Fuzzy Set (CIFS) was given by Garg and Rani [39] in a multiple-criteria decision-making for uncertain and vague data. The quaternion representation and distance on CIFS were proposed in [40] with an application in medical diagnosis. These formulas of quaternion representation and distance measure were used in a diagnosis model by calculating patient-disease relations. The threshold obtained via learning process was used to decide the output of model. In the Interval-Valued Complex Fuzzy Set (IvCFS), distance measures were defined on Euclidean metric and Hamming metric [37]. The authors presented an example to illustrate the use of these measures in decision-making. In the case of Complex Neutrosophic Set (CNS), similarity measures with the weighted versions were introduced in [32]. Using these measures, the decision-making model could rank the priority of candidates. The best one was selected to make a decision. These measures lead to good decisions because they considered the interaction among attributes in the dataset and the indeterminacy of data.

\subsection{Fuzzy Inference System in Complex Fuzzy Set}

Many intelligent systems with different applications were based on FIS [2-4,6,7]. Sagir et al. [4] proposed two extended models of ANFIS to apply to the heart disease prediction problem. The limitations of these extended models are that the accuracy of classification is not very high and the number of rules is great. A combination of multiple kernel learning and ANFIS was introduced by Manogaran and Varatharajan [7]. This method resulted in higher performance than other compared models. However, the application of this model focused on diagnosing heart disease with only two input features. ANFIS was also used in detecting lung cancer [3] by cancerous and non-cancerous segmentation on computed tomography images.

ANCFIS in time series forecasting has higher quality than other related models, including ANFIS [25]. Using the experimental results on five real datasets, the values of MSE and NDEI from applying ANCFIS are less than those of the compared models. ANCFIS was improved as FANCFIS to deal with multivariate time series problem $[27,41]$. This model was designed to maintain the accuracy and decrease time computing of ANCFIS. Many applications of these systems were also presented in $[24,26,42]$. The combination of the CFS and machine learning or other state-of-the-art tools was one of the popular approaches to carry out practical problems. In [43], a CFS with multiswarm learning was proposed for multiclass prediction problems. Granular computing was utilized for complex fuzzy sets in [44]. The granulation was used to interpret complex fuzzy contexts provided by users. 


\section{Preliminaries}

\subsection{Complex Fuzzy Set}

Definition 1 [1]. A fuzzy set F over $X$ is defined by

$$
F=\left\{\left(x, \mu_{F}(x)\right): x \in X\right\}
$$

where the membership function of $F, \mu_{F}(x)$, is $\mu_{F}: X \rightarrow[0,1]$. For each $x \in X$, the value $\mu_{F}(x)$ represents the degree of membership of $x$ in the fuzzy set $F$.

Definition 2 [15]. A complex fuzzy set (CFS) S on X, is characterized by a membership function $\eta_{S}(x)$ that lies within the unit circle in the complex plane and has the form $p_{S}(x) \cdot e^{i \cdot \mu_{S}(x)}$ where the amplitude $p_{S}(x)$ and phase $\mu_{S}(x)$ are both real-valued, $p_{S}(x) \in[0,1]$, and $i=\sqrt{-1}$.

$$
S=\left\{x, \eta_{S}(x) \mid x \in X\right\}
$$

Definition 3 [45]. Some basic operations of CFS:

Consider two CFSs, $A$ and $B$, in a universe of discourse $X$ with membership degrees of $\eta_{A}(x)=$ $p_{A}(x) e^{j \mu_{A}(x)}, \eta_{B}(x)=p_{B}(x) e^{j \mu_{B}(x)}$, respectively. The operations of these two CFSs are defined as follows:

$$
\begin{aligned}
& \eta_{A \cup B}(x)=\left[p_{A}(x) \oplus p_{B}(x)\right] e^{j\left(\mu_{A \cup B}\right)}=\max \left(p_{A}(x), p_{B}(x)\right) \cdot e^{j\left(\max \left(\mu_{A}(x), \mu_{B}(x)\right)\right)} \\
& \eta_{A \cap B}(x)=\left[p_{A}(x) * p_{B}(x)\right] e^{j\left(\mu_{A \cap B}\right)}=\min \left(p_{A}(x), p_{B}(x)\right) \cdot e^{j\left(\min \left(\mu_{A}(x), \mu_{B}(x)\right)\right)}
\end{aligned}
$$

where $\eta_{A \cup B}(x)$ is the union and $\eta_{A \cap B}(x)$ is the intersection operation of the CFSs $A$ and $B, *$ is t-norm, and $\oplus$ is $\mathrm{t}$-conorm.

Definition 4 [45]. Let A be a complex fuzzy set on $X$. The complement of $S$ is

$$
\eta_{c(A)}(x)=p_{c(A)}(x) e^{j \mu_{c(A)}(x)}=\left(1-p_{c(A)}\right)(x) \cdot e^{j\left(2 \pi-\mu_{c(A)}(x)\right)}
$$

Definition 5 [45]. Let $A$ and $B$ be two CFSs on $X$ with $\eta_{A}(x)=p_{A}(x) e^{j \mu_{A}(x)}, \eta_{B}(x)=p_{B}(x) e^{j \mu_{B}(x)}$. The complex fuzzy product of $A$ and $B$ is

$$
\eta_{A^{\circ} B}(x)=p_{A^{\circ} B}(x) e^{j \mu_{A^{\circ} B}(x)}=\left(p_{A}(x) \cdot p_{B}(x)\right) \cdot e^{j \frac{\mu_{A}(x) \cdot \mu_{B}(x)}{2 \pi}}
$$

Definition 6 [45]. A distance of complex fuzzy sets is $\rho:\left(F^{*}(U) \times F^{*}(U)\right) \rightarrow[0,1]$, for any $A, B$, and $C \in$ $F^{*}(U)$

i. $\rho(A, B) \geq 0, \rho(A, B)=0 \operatorname{IFF} A=B$,

ii. $\rho(A, B)=\rho(B, A)$,

iii. $\rho(A, B) \leq \rho(A, C)+\rho(C, B)$,

where $F^{*}(U)$ is the set of all complex fuzzy sets in $U$.

Definition 7 [45]. Assume $A$ and $B$, with $\eta_{A}(x)=p_{A}(x) e^{j \mu_{A}(x)}, \eta_{B}(x)=p_{B}(x) e^{j \mu_{B}(x)}$. A and $B$ are $\delta$-equal $\operatorname{IFF}(A, B) \leq 1-\delta, 0 \leq \delta \leq 1$. 
Definition 8 [45]. Assume $A$ and $B$, with $\eta_{A}(x)=p_{A}(x) e^{j \mu_{A}(x)}, \eta_{B}(x)=p_{B}(x) e^{j \mu_{B}(x)}$. The distance of two CFSs defined on the same product space is defined as follows:

$$
d(A, B)=\max \left(\sup _{(x, y) \in U x V}\left|p_{A}(x, y)-p_{B}(x, y)\right|, \frac{1}{2 \pi} \sup _{(x, y) \in U x V}\left|\mu_{A}(x, y)-\mu_{B}(x, y)\right|\right)
$$

\subsection{Mamdani Complex Fuzzy Inference System (M-CFIS)}

In this section, we introduce the Mamdani Complex Fuzzy Inference System (M-CFIS) [23]. The general structure of Mamdani CFIS consists of six stages [23]:

Let $x_{1}, x_{2}, \ldots, x_{n} \in \mathbb{C}$ be the inputs of this model.

Stage 1: Establish a set of complex fuzzy rules

Based on practical application, we will determine the set of complex fuzzy rules in the form:

$$
\begin{gathered}
\mathrm{CFR}_{1}: \text { If } x_{1,1} \text { is } A_{1,1} \mathbf{O}_{1,1} x_{1,2} \text { is } A_{1,2} \mathbf{O}_{1,2} \ldots \mathbf{O}_{1, n_{1}-1} x_{1, n_{1}} \text { is } A_{1, n_{1}} \text {, is } Z_{1} \\
\mathrm{CFR}_{2}: \text { If } x_{2,1} \text { is } A_{2,1} \mathbf{O}_{2,1} x_{2,2} \text { is } A_{2,2} \mathbf{O}_{2,2} \ldots \mathbf{O}_{2, n_{2}-1} x_{2, n_{2}} \text { is } A_{2, n_{2}} \text {, is } Z_{2} \\
\ldots \\
\ldots \\
\mathrm{CFR}_{\mathbf{k}}: \text { If } x_{k, 1} \text { is } A_{k, 1} \mathbf{O}_{k, 1} x_{k, 2} \text { is } A_{k, 2} \mathbf{O}_{k, 2} \ldots \mathbf{O}_{k, n_{k}-1} x_{k, n_{k}} \text { is } A_{k, n_{k}} \text {, is } Z_{k}
\end{gathered}
$$

With all $u, v$ :

(i) $(u, v) \in\{1,2, \ldots, n\}$, with $1 \leq u, 1<u, 2<\ldots<u, n_{u} \leq n$;

(ii) $\eta_{A_{u, v}}\left(x_{u, v}\right)=p_{A_{u, v}}\left(x_{u, v}\right) \mathrm{e}^{j \mu_{A_{u, v}}\left(x_{u, v}\right)}$, with $p_{A_{u, v}}: \mathbb{C} \rightarrow[0,1]$ and $\mu_{A_{u, v}}: \mathbb{C} \rightarrow(0,2 \pi]$;

(iii) $\eta_{Z_{u}}(y)=p_{Z_{u}}(y) \mathrm{e}^{j \mu_{Z_{u}}(y)}$, with $p_{Z_{u}}: \mathbb{C} \rightarrow[0,1]$ and $\mu_{Z_{u}}: \mathbb{C} \rightarrow(0,2 \pi]$;

(iv) $T_{0}$ is a T-norm, and $S_{0}$ is the $\mathrm{S}$-norm (i.e., the T-conorm) that corresponds to $T_{0}$;

(v) $\mathbf{O}_{\mathrm{u}, \mathrm{v}}=$ and IFF $\mathrm{N}_{\mathrm{u}, \mathrm{v}}=T_{0}$;

(vi) $\mathbf{O}_{\mathrm{u}, \mathrm{v}}=$ or IFF $\mathrm{N}_{\mathrm{u}, \mathrm{v}}=S_{0}$.

Stage 2: Fuzzification of the inputs

Inputs are fuzzified using complex membership function $\eta(x)=p(x) \cdot e^{j} \mu(x)$, where $\mu(x) \in(0,2 \pi]$, $p(x) \in[0,1]$, and $p(x)$ and $\mu(x)$ represent the amplitude and phase terms of the elements, respectively.

Stage 3: Establish the firing strength of rule

This stage computes the firing strength $\omega_{u}$ for each complex fuzzy rule as: $\omega_{u}=\tau_{u} \mathrm{e}^{j \psi_{u}}$.

Stage 4: Calculate the consequence of the complex fuzzy rules

In Mamdani CFIS, the value of the consequence of the complex fuzzy rules is obtained by using the Mamdani implication rule.

$$
\eta_{A \rightarrow B}(x, y)=\left(p_{A}(x) \cdot p_{B}(y)\right) \cdot e^{j 2 \pi\left(\frac{\mu_{A}(x)}{2 \pi} \cdot \frac{\mu_{B}(y)}{2 \pi}\right)}
$$

Choose a function $U_{0}:[0,1]^{2} \rightarrow[0,1]$, with $U_{0}(1,1)=1$, and a function $g_{0}:(0,2 \pi]^{2} \rightarrow(0,2 \pi]$, with $g_{0}(2 \pi, 2 \pi)=2 \pi$. We form the consequent of $\mathrm{CFR}_{u}$ for each $u$ :

$$
\Gamma_{u}(y)=U_{0}\left(\tau_{u}, r_{C_{u}}(y)\right) \mathrm{e}^{j g_{0}\left(\psi_{u}, \mu_{C_{u}}(y)\right)}=\omega_{u} \cdot \eta_{C_{u}}(y)
$$

where "." denotes the complex dot product.

Stage 5: Aggregation

In this stage, the output distribution is calculated as follows:

$$
D(y)=\Gamma_{1}(y)+\Gamma_{2}(y)+\ldots+\Gamma_{k}(y) .
$$

Stage 6: Defuzzification. 
Choose a function $\Phi: \mathcal{F}(\mathbb{C}, \mathbb{C}) \rightarrow \mathbb{C}$. Determine the value of the output $y_{\text {op }}=\Phi(D)$. For example, we can choose the trapezoidal approximation such as $\Phi(D)=\frac{\int_{-\infty}^{\infty} y|D(y)| \mathrm{d} y}{\int_{-\infty}^{\infty}|D(y)| \mathrm{d} y}$.

\subsection{Granular Computing}

Granular computing [46,47] generally refers information granulation that includes probabilistic set, fuzzy set, and rough set. In the context of fuzzy sets, each element can be viewed as a granule of a certain degree of membership to the set. It is used to simplify complex problems by decomposing strategy in term of information granulation. Studying rule learning with granular computing has an important role in improving the model accuracy. Thus, the relationship between granular computing and rule-based systems is argued.

\section{Proposed M-CFIS-R System}

\subsection{Main Ideas}

It has been observed from Section 2 that changing the number of rules in a rule base for better performance of classification is still a challenge when designing a FIS model. Hence, it is necessary to have an effective measure to evaluate the importance of each rule in the rule base. This section will propose three similarity measures in the Training stage. In our decision-making model, granular computing is used in the last stage of Training. The purpose of using granular computing is to reduce the rules with high similarity or to add more rules in order to get higher coverage. The result of this stage is a new rule set with suitable number of rules with high classification accuracy compared to the original rule set. Comparing with the architecture of M-CFIS [23], we add the Training process in order to create the original complex fuzzy rule base and improve it by the Granular Computing with Complex Fuzzy Measure (i.e., Granular Complex Fuzzy Measure). The Testing phase follows the inference process in the M-CFIS discussed in Section 3.2 but with the reduced complex fuzzy base. The model is divided into two parts: (i) Training used to train the generation of fuzzy rules is discussed in detail in Section 4.2; (ii) Testing used to test the performance of the rule system is discussed in Section 4.3.

\subsection{Training}

In this model, we divide the dataset into the Training-Validation-Testing parts by K-Folds (where $\mathrm{K}$ is often small, e.g., 3). From the Training data, we build the real and imaginary data (presented in Section 4.2.1). Then, fuzzy clustering (i.e., Fuzzy C-Means [48]) is performed for each attribute of those data to obtain the set of fuzzy rules, which is considered as the original complex fuzzy rule base (see Section 4.2.2). This rule base is evaluated on the Validation data to get the performance, namely $A$ (see Section 4.2.3). Next, we try to improve the original complex fuzzy rule base by calculating the correlations between complex fuzzy rules based on different new complex fuzzy measures (see Section 4.2.4). The similarities of complex fuzzy rules are finally determined by granular computing according to each label of Validation data (see Section 4.2.5). We then evaluate performance of the new complex fuzzy rule base called $A^{\prime}$ on the Validation data by the same inference module (similar to Section 4.2.3). If $A^{\prime}$ is better than $A$, we end the Training and proceed to Testing; otherwise, we repeat the process of using Granular Complex Fuzzy Measure to retrain. The Training process can be seen in Figure 1. 


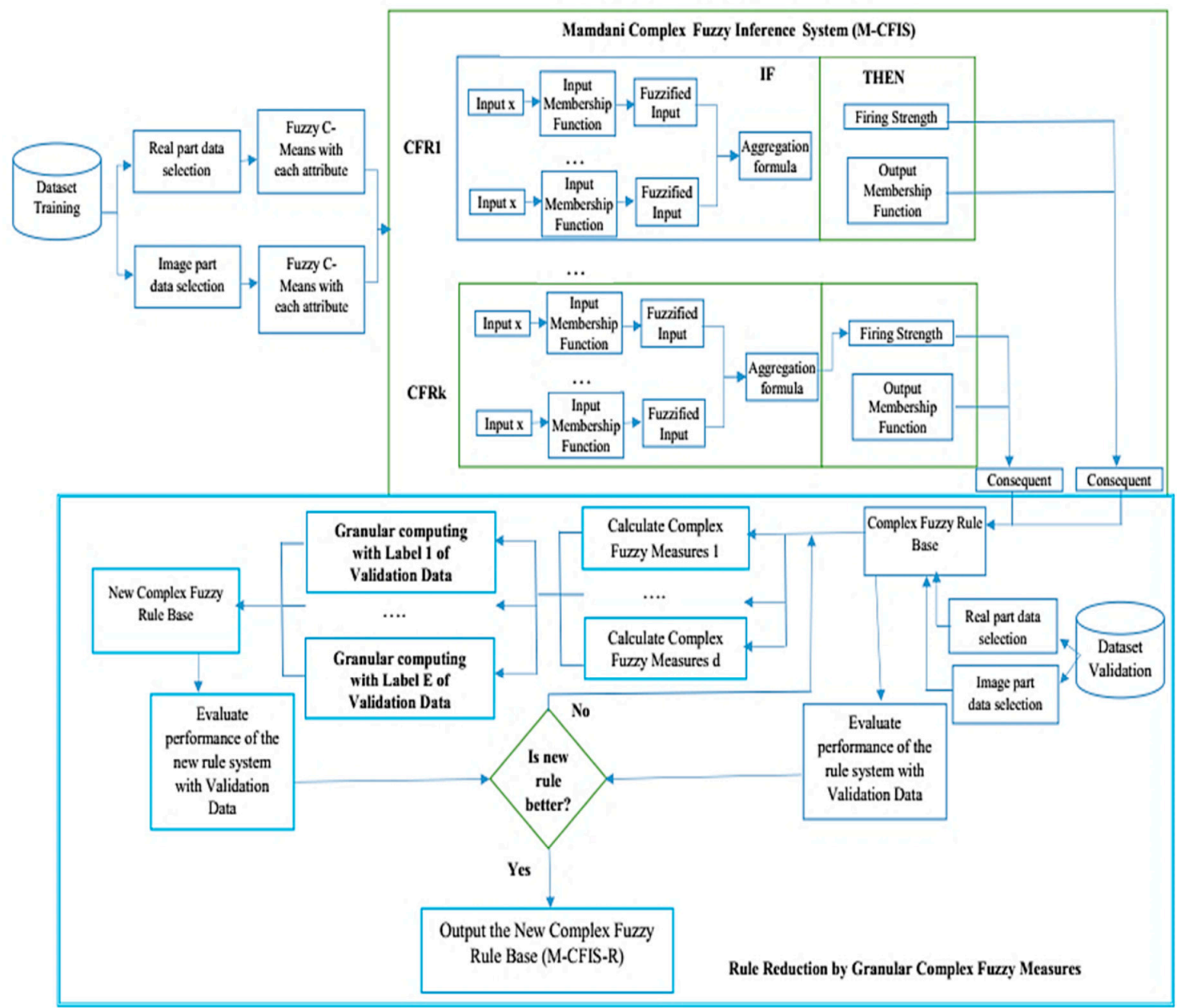

Figure 1. Training diagram for the proposed model.

\subsubsection{Real and Imaginary Data Selection}

From the Training data, we build the real and imaginary data as follows: The real data are defined as the original data values. The imaginary data at record $P$ of attribute $Q$ is determined as var. $P($ row $)+$ var. $Q$ (column), where var.P (row) is the variance in row at row $P$ and var. $Q$ (column) is the variance according to the column in column $Q$.

\subsubsection{Fuzzy C-Means (FCM)}

In this study, we use the Fuzzy C-Means clustering method (Algorithm 1) for dividing the data according to each attribute into several groups. The number of clusters specified for each attribute is different based on the semantic value of the attribute. The number of clusters of an attribute in the real and imaginary parts is the same. Finally, we produce complex fuzzy rules from each cluster. 


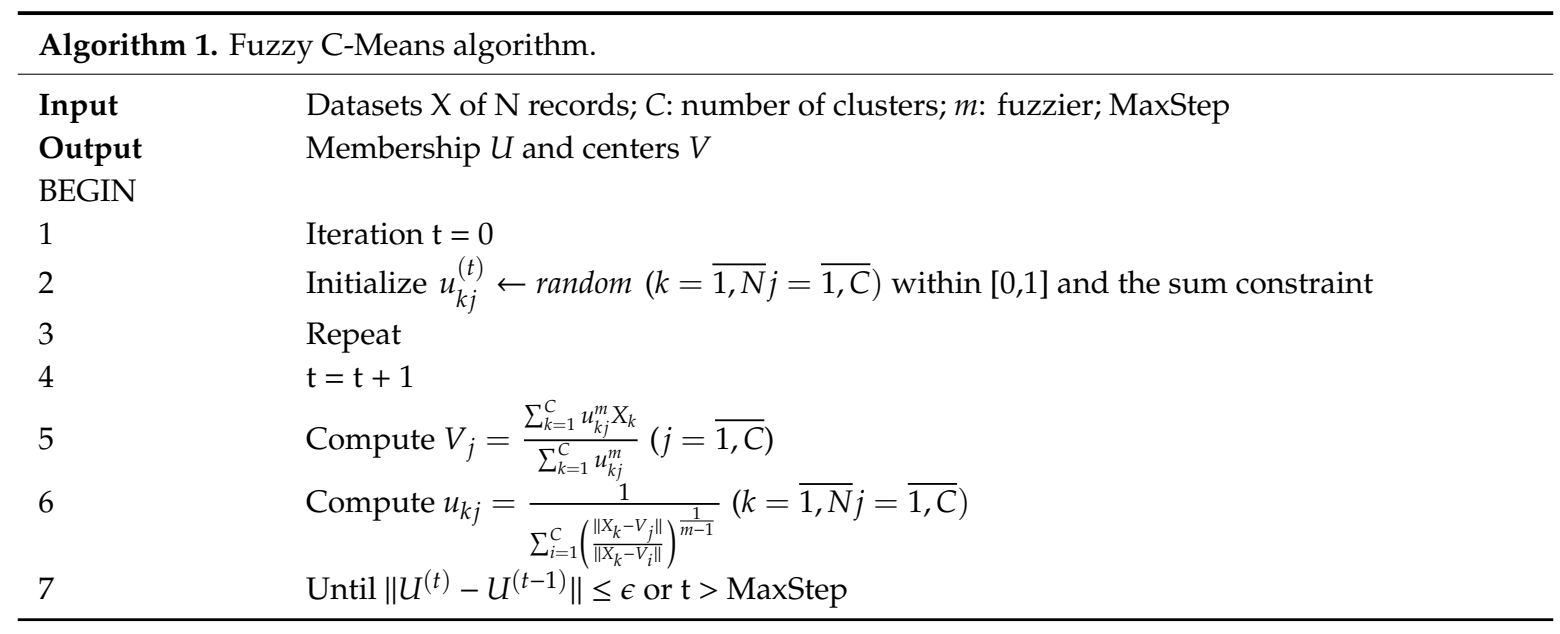

4.2.3. Evaluating Performance of the Rule-Based System

After obtaining the complex fuzzy rule base, we use the Validation data to derive the outputs and evaluate the performance through Accuracy, Precision, and Recall.

$$
\text { Accuracy }=\frac{\mathrm{TN}+\mathrm{TP}}{T N+\mathrm{FN}+\mathrm{FP}+\mathrm{TP}}
$$

Recall $=\frac{|T P|}{|T P|+|F N|}$

Precision $=\frac{|T P|}{|T P|+|F P|}$

\subsubsection{Complex Fuzzy Measures}

In this section, we propose three complex fuzzy similarity measures with their weighted versions as below.

Complex Fuzzy Cosine Similarity Measure (CFCSM)

Definition 9. Assume that there are two complex fuzzy sets, namely $S_{1}=r_{S_{1}}(x) e^{j \mu_{S_{1}}(x)}$ and $S_{2}=r_{S_{2}}(x) e^{j \mu_{S_{2}}(x)}$, $x \in X$. A Complex Fuzzy Cosine Similarity Measure (CFCSM) between $S_{1}$ and $S_{2}$ is

$$
\mathrm{C}_{\mathrm{CFS}}=\frac{1}{\mathrm{n}} \sum_{\mathrm{j}=1}^{\mathrm{n}} \frac{\left(\mathrm{a}_{1} \mathrm{~b}_{1}\right)\left(\mathrm{a}_{2} \mathrm{~b}_{2}\right)}{\sqrt{\left(\mathrm{a}_{1} \mathrm{~b}_{1}\right)^{2}}+\sqrt{\left(\mathrm{a}_{2} \mathrm{~b}_{2}\right)^{2}}}
$$

where

$$
\begin{gathered}
a_{1}=\operatorname{Re}\left(p_{S_{1}}(x) e^{j \mu_{S_{1}}(x)}\right), b_{1}=\operatorname{Im}\left(p_{S_{1}}(x) e^{j \mu_{S_{1}}(x)}\right), a_{2}=\operatorname{Re}\left(p_{S_{2}}(x) e^{j \mu_{S_{2}}(x)}\right), \\
b_{2}=\operatorname{Im}\left(p_{S_{2}}(x) e^{j \mu_{S_{2}}(x)}\right)
\end{gathered}
$$

Proposition 1. Let $S_{1}$ and $S_{2}$ be complex fuzzy sets. Then,

1. $0 \leq C_{C F S}\left(S_{1}, S_{2}\right) \leq 1$;

2. $C_{C F S}\left(S_{1}, S_{2}\right)=C_{C F S}\left(S_{2}, S_{1}\right)$;

3. $C_{C F S}\left(S_{1}, S_{2}\right)=1$ if and only if $S_{1}=S_{2}$;

4. If $S_{1} \subset S_{2} \subset S$ then $C_{C F S}\left(S_{1}, S\right) \leq C_{C F S}\left(S_{1}, S_{2}\right)$ and $C_{C F S}\left(S_{1}, S\right) \leq C_{C F S}\left(S_{2}, S\right)$.

\section{Proof.}

1. It is correct because all positive values of cosine function are within 0 and 1 .

2. Trivial. 
3. When $S_{1}=S_{2}$ then obviously $C_{C F S}\left(S_{1}, S_{2}\right)=1$. If $C_{C F S}\left(S_{1}, S_{2}\right)=1, a_{1}=a_{2}, b_{1}=b_{2}$. This implies that $S_{1}=S_{2}$.

4. Let $S=\left\langle p_{S}(x) \cdot e^{j \mu_{S}(x)}\right\rangle$ and also assume that $L_{1}=\operatorname{Re}\left[p_{S}(x) \cdot e^{j \mu_{S}(x)}\right]$ and $L_{2}=\operatorname{Im}\left[p_{S}(x) \cdot e^{j \mu_{S}(x)}\right]$. If $S_{1} \subset S_{2} \subset S$, we can write that $a_{1} b_{1} \leq a_{2} b_{2} \leq L_{1} L_{2}$. The cosine function is a decreasing function within the interval $\left[0, \frac{\pi}{2}\right]$. Then, we can write $C_{C F S}\left(S_{1}, S\right) \leq C_{C F S}\left(S_{1}, S_{2}\right)$ and $C_{C F S}\left(S_{1}, S\right) \leq$ $C_{C F S}\left(S_{2}, S\right)$.

Definition 10. Weighted Complex Fuzzy Cosine Similarity Measure (WCNCSM).

Assume that there are two complex fuzzy sets, namely $S_{1}=p_{S_{1}}(x) e^{j \mu_{S_{1}}(x)}$ and $S_{2}=p_{S_{2}}(x) e^{j \mu_{S_{2}}(x)}$, $\mathrm{x} \in \mathrm{X}$. A Weighted Complex Fuzzy Cosine Similarity Measure between $S_{1}$ and $S_{2}$ is

$$
C_{W C F S}=\sum_{j=1}^{n} w_{j}\left[\frac{\sqrt{a_{1} b_{1} a_{2} b_{2}}}{\sqrt{a_{1} b_{1}}+\sqrt{a_{2} b_{2}}}\right] \text { where } \sum_{j=1}^{n} w_{j}=1
$$

Complex Fuzzy Dice Similarity Measure (CFDSM)

Definition 11. Assume that there are two complex fuzzy sets namely $S_{1}=r_{S_{1}}(x) e^{j \mu_{S_{1}}(x)}$ and $S_{2}=$ $r_{S_{2}}(x) e^{j \mu_{S_{2}}(x)}, x \in X$. A Complex Fuzzy Dice Similarity Measure (CFDSM) between $S_{1}$ and $S_{2}$ is

$$
D_{C F S}=\frac{1}{n} \sum_{j=1}^{n} \frac{2 \sqrt{a_{1} b_{1} a_{2} b_{2}}}{a_{1} b_{1}+a_{2} b_{2}}
$$

where

$$
\begin{gathered}
a_{1}=\operatorname{Re}\left(p_{S_{1}}(x) e^{j \mu_{S_{1}}(x)}\right), b_{1}=\operatorname{Im}\left(p_{S_{1}}(x) e^{j \mu_{S_{1}}(x)}\right), a_{2}=\operatorname{Re}\left(p_{S_{2}}(x) e^{j \mu_{S_{2}}(x)}\right) \\
b_{2}=\operatorname{Im}\left(p_{S_{2}}(x) e^{j \mu_{S_{2}}(x)}\right) .
\end{gathered}
$$

Proposition 2. Let $S_{1}$ and $S_{2}$ be complex fuzzy sets. Then,

1. $0 \leq D_{C F S}\left(S_{1}, S_{2}\right) \leq 1$;

2. $D_{C F S}\left(S_{1}, S_{2}\right)=D_{C F S}\left(S_{2}, S_{1}\right)$;

3. $D_{C F S}\left(S_{1}, S_{2}\right)=1$ if and only if $S_{1}=S_{2}$;

4. If $S_{1} \subset S_{2} \subset S$ then $D_{C F S}\left(S_{1}, S\right) \leq D_{C F S}\left(S_{1}, S_{2}\right)$ and $D_{C F S}\left(S_{1}, S\right) \leq D_{C F S}\left(S_{2}, S\right)$.

Proof. The proof is similar to Proposition 1.

Definition 12. Weighted Complex Fuzzy Dice Similarity Measure (WCFDSM).

Assume that there are two complex fuzzy sets, namely $S_{1}=p_{S_{1}}(x) e^{j \mu_{S_{1}}(x)}$ and $S_{2}=p_{S_{2}}(x) e^{j \mu_{S_{2}}(x)}$, $x \in X$. A Weighted Complex Fuzzy Dice Similarity Measure between $S_{1}$ and $S_{2}$ is

$$
D_{W C N S}=\sum_{j=1}^{n} w_{j}\left[\frac{2\left(\sqrt{a_{1} b_{1} a_{2} b_{2}}\right)}{\sqrt{a_{1} b_{1}}+\sqrt{a_{2} b_{2}}}\right] \text { where } \sum_{j=1}^{n} w_{j}=1
$$


Complex Fuzzy Jaccard Similarity Measure (CFJSM)

Definition 13. Assume that there are two complex fuzzy sets, namely $S_{1}=p_{S_{1}}(x) e^{j \mu_{S_{1}}(x)}$ and $S_{2}=$ $p_{S_{2}}(x) e^{j \mu_{S_{2}}(x)}, x \in X$. A Complex Fuzzy Jaccard Similarity Measure (CFJSM) between $S_{1}$ and $S_{2}$ is

$$
J_{C F S}=\frac{1}{n} \sum_{j=1}^{n} \frac{\sqrt{a_{1} b_{1} a_{2} b_{2}}}{\left(a_{1} b_{1}+a_{2} b_{2}\right)-\left(\sqrt{a_{1} b_{1}}+\sqrt{a_{2} b_{2}}\right)}
$$

where $a_{1}=\operatorname{Re}\left(p_{S_{1}}(x) e^{j \mu_{S_{1}}(x)}\right), b_{1}=\operatorname{Im}\left(p_{S_{1}}(x) e^{j \mu_{S_{1}}(x)}\right), a_{2}=\operatorname{Re}\left(p_{S_{2}}(x) e^{j \mu_{S_{2}}(x)}\right), b_{2}=\operatorname{Im}\left(p_{S_{2}}(x) e^{j \mu_{S_{2}}(x)}\right)$.

Proposition 3. Let $S_{1}$ and $S_{2}$ be complex fuzzy sets. Then,

1. $0 \leq J_{C F S}\left(S_{1}, S_{2}\right) \leq 1$;

2. $J_{C F S}\left(S_{1}, S_{2}\right)=J_{C F S}\left(S_{2}, S_{1}\right)$;

3. $J_{C F S}\left(S_{1}, S_{2}\right)=1$ if and only if $S_{1}=S_{2}$;

4. If $S_{1} \subset S_{2} \subset S$ then $J_{C F S}\left(S_{1}, S\right) \leq J_{C F S}\left(S_{1}, S_{2}\right)$ and $J_{C F S}\left(S_{1}, S\right) \leq J_{C F S}\left(S_{2}, S\right)$.

Proof. The proof is similar to Proposition 1.

Definition 14. Weighted Complex Fuzzy Jaccard Similarity Measure (WCFJSM)

Assume that there are two complex fuzzy sets namely $S_{1}=p_{S_{1}}(x) e^{j \mu_{S_{1}}(x)}$ and $S_{2}=p_{S_{2}}(x) e^{j \mu_{S_{2}}(x)}$, $x \in X$. A Weighted Complex Fuzzy Dice Similarity Measure between $S_{1}$ and $S_{2}$ is

$$
J_{\text {WCNS }}=\sum_{j=1}^{n} w_{j}\left[\frac{\sqrt{a_{1} b_{1} a_{2} b_{2}}}{\sqrt{a_{1} b_{1}}-\sqrt{a_{2} b_{2}}}\right] \text { where } \sum_{j=1}^{n} w_{j}=1
$$

\subsubsection{Granular Complex Fuzzy Measures}

In this section, we describe how to determine the final similarity between complex fuzzy rules from the correlations of rules described in Section 4.2.4. To accomplish this, we introduce an idea of granular computing to conceptualize relationships for a combination of fuzzy correlation measures. Assume that the outputs of three similarity measures in Section 4.2.4 are three corresponding squared matrices whose elements are the correlations between pairs of complex fuzzy rules: $D^{1}, D^{2}, D^{3}$. We determine the final degree of similarity between complex fuzzy rules based on the aggregation:

$$
F_{i j}=a_{1} D_{i j}^{1}+a_{2} D_{i j}^{2}+a_{3} D_{i j}^{3}
$$

For each set of labels, e.g., label 1 , we obtain $F_{i j}(l)$ to be determined $a_{1}(l), a_{2}(l), \ldots a_{e}(l)$.

$$
a_{t}(l)=\sum_{i=1}^{\left|D^{t} / l\right|} \sum_{j=i+1}^{\left|D^{t} / l\right|} \frac{\left(D_{i j}^{t} / t\right)}{\left|D^{t} / l\right|}
$$

For rules other than labels, then $F_{i j}=0$. From these, we obtain the matrix $F$.

A new complex fuzzy rule base is found from $F$ by removing rules having a high or maximal degree of similarity within a group. Then, we proceed to the next steps to evaluate the performance of the new rule system. In cases that the performance of the new complex fuzzy rule base is worse than that of the current rule, we return to the steps of computing the complex fuzzy measures (Section 4.2.4) and granular computing (Section 4.2.5) for the new complex fuzzy rule base. The iteration stops either when the performance of the new complex fuzzy rule base is better than that of the current base or the 
cardinality of rules according to any label is equal to 1 . The following example demonstrates the main activities of granular computing in M-CFIS-R.

Example 1. Suppose we have a set of 6 complex fuzzy rules in which 3 rules $R_{1}, R_{3}, R_{4}$ have label $1(k=1)$, and 3 rules $R_{2}, R_{5}, R_{6}$ have label 2:

$R_{1}$ : If $x_{1}$ is Medium and $x_{2}$ is High and $x_{3}$ is High then $k$ is 1

$R_{2}$ : If $x_{1}$ is High and $x_{2}$ is Low and $x_{3}$ is Low then $k$ is 2

$R_{3}$ : If $x_{1}$ is Low and $x_{2}$ is Medium and $x_{3}$ is High then $k$ is 1

$R_{4}$ : If $x_{1}$ is Low and $x_{2}$ is High and $x_{3}$ is Medium then $k$ is 1

$R_{5}$ : If $x_{1}$ is High and $x_{2}$ is Low and $x_{3}$ is Medium then $k$ is 2

$R_{6}$ : If $x_{1}$ is Medium and $x_{2}$ is Low and $x_{3}$ is Low then $k$ is 2

Using the complex fuzzy measures (Section 4.2.4), we obtain three matrices as follows:

$$
\begin{aligned}
& D^{1}=\left[\begin{array}{cccccc}
0 & 0.5 & 0.8 & 0.7 & 0.4 & 0.3 \\
0.5 & 0 & 0.5 & 0.4 & 0.8 & 0.9 \\
0.8 & 0.5 & 0 & 0.9 & 0.4 & 0.5 \\
0.7 & 0.4 & 0.9 & 0 & 0.5 & 0.3 \\
0.4 & 0.8 & 0.4 & 0.5 & 0 & 0.7 \\
0.3 & 0.9 & 0.5 & 0.3 & 0.7 & 0
\end{array}\right] \\
& D^{2}=\left[\begin{array}{cccccc}
0 & 0.2 & 0.5 & 0.4 & 0.2 & 0.1 \\
0.2 & 0 & 0.2 & 0.3 & 0.5 & 0.6 \\
0.5 & 0.2 & 0 & 0.5 & 0.2 & 0.1 \\
0.4 & 0.3 & 0.5 & 0 & 0.2 & 0.3 \\
0.2 & 0.5 & 0.2 & 0.2 & 0 & 0.5 \\
0.1 & 0.6 & 0.1 & 0.3 & 0.5 & 0
\end{array}\right] \\
& D^{3}=\left[\begin{array}{cccccc}
0 & 0.1 & 0.4 & 0.4 & 0.2 & 0.1 \\
0.1 & 0 & 0.1 & 0.2 & 0.4 & 0.3 \\
0.4 & 0.1 & 0 & 0.4 & 0.1 & 0.2 \\
0.4 & 0.2 & 0.4 & 0 & 0.2 & 0.1 \\
0.2 & 0.4 & 0.1 & 0.2 & 0 & 0.3 \\
0.1 & 0.3 & 0.2 & 0.1 & 0.3 & 0
\end{array}\right]
\end{aligned}
$$

We calculate the coefficients of each matrix according to the labels 1, 2:

$$
\begin{aligned}
& a_{1}^{1}=\frac{0.8+0.7+0.9}{3}=0.8 a_{2}^{1}=\frac{0.5+0.4+0.5}{3}=0.467 a_{3}^{1}=\frac{0.4+0.4+0.4}{3}=0.4 \\
& a_{1}^{2}=\frac{0.8+0.9+0.7}{3}=0.8 a_{2}^{2}=\frac{0.5+0.6+0.5}{3}=0.533 a_{3}^{1}=\frac{0.4+0.3+0.3}{3}=0.333
\end{aligned}
$$

We calculate the matrix $F$ as follows:

$$
F=\left[\begin{array}{cccccc}
0 & 0 & 1.034 & 0.907 & 0 & 0 \\
0 & 0 & 0 & 0 & 1.04 & 1.14 \\
0.397 & 0 & 0 & 1.114 & 0 & 0 \\
0.563 & 0 & 0.51 & 0 & 0 & 0 \\
0 & 0.29 & 0 & 0 & 0 & 0.926 \\
0 & 0.657 & 0 & 0 & 0.926 & 0
\end{array}\right]
$$


It is obvious that the rules with highest similarity within label 1 are $R_{3}$ and $R_{4}$, and the rules with the highest similarity within label 2 are and $R_{2}$ and $R_{6}$. Then, the new complex fuzzy rule base is: $R_{1}$, $R_{3}$ with label $1 ; R_{2}, R_{5}$ with label 2.

We calculate performance of the new rule base. If it is worse, we return to compute the complex fuzzy measures with the current rule base including $R_{1}, R_{3}$ with label 1 and $R_{2}, R_{5}$ with label 2 . Here, we demonstrate Iteration 2 .

The second iteration: The second set of fuzzy rules $R^{\prime}$ is: $R_{1}^{\prime}, R_{3}^{\prime}$ have label $1, R_{2}^{\prime}, R_{4}^{\prime}$ have label 2. For clarity, we assign a mark (') to differentiate between iterations. The corresponding measure values are:

$$
\begin{aligned}
D^{1^{\prime}} & =\left[\begin{array}{cccc}
0 & 0.5 & 0.8 & 0.3 \\
0.5 & 0 & 0.4 & 0.7 \\
0.8 & 0.4 & 0 & 0.3 \\
0.3 & 0.7 & 0.3 & 0
\end{array}\right] \\
D^{2^{\prime}} & =\left[\begin{array}{cccc}
0 & 0.2 & 0.5 & 0.1 \\
0.2 & 0 & 0.2 & 0.4 \\
0.5 & 0.2 & 0 & 0.1 \\
0.1 & 0.4 & 0.1 & 0
\end{array}\right] \\
D^{3^{\prime}} & =\left[\begin{array}{cccc}
0 & 0.1 & 0.3 & 0.1 \\
0.1 & 0 & 0.1 & 0.4 \\
0.3 & 0.1 & 0 & 0.2 \\
0.1 & 0.4 & 0.2 & 0
\end{array}\right]
\end{aligned}
$$

We calculate the coefficients of each matrix according to the labels 1 and 2:

$$
\begin{aligned}
& a_{1}^{\prime 1}=\frac{0.8}{1}=0.8 a_{2}^{\prime 1}=\frac{0.5}{1}=0.5 a_{3}^{\prime 1}=\frac{0.3}{1}=0.3 \\
& a_{1}^{\prime 2}=\frac{0.7}{1}=0.7 a_{2}^{\prime 2}=\frac{0.4}{1}=0.4 a_{3}^{\prime 1}=\frac{0.4}{1}=0.4
\end{aligned}
$$

We calculate the matrix $F$ as follows:

$$
F^{\prime}=\left[\begin{array}{cccc}
0 & 0 & 0.98 & 0 \\
0 & 0 & 0 & 0.81 \\
0.98 & 0 & 0 & 0 \\
0 & 0.81 & 0 & 0
\end{array}\right]
$$

We define the similarity with the same label and determine the highest similarity. The following rules are similar: $R_{1}^{\prime}$ and $R_{3}^{\prime}, R_{2}^{\prime}$, and $R_{4}^{\prime}$. Then, the new rule base is: $R_{1}^{\prime}$ with label $1 ; R_{2}^{\prime}$ with label 2. We continue to compute performance of the new rule base. Even if the performance is not better than that of the current rule base, we still stop the algorithm because the cardinality of rules in both labels 1 and 2 is 1 . In order to obtain the best performance, we may use the original complex fuzzy rule base generated from Training as the final results. As a result, the proposed M-CFIS-R at least has performance equal to M-CFIS in the worst case.

\subsection{Testing}

We perform a similar procedure with M-CFIS [23] for testing the performance of the system with the reduced complex fuzzy rule base found in the Training phase (Figure 2). 


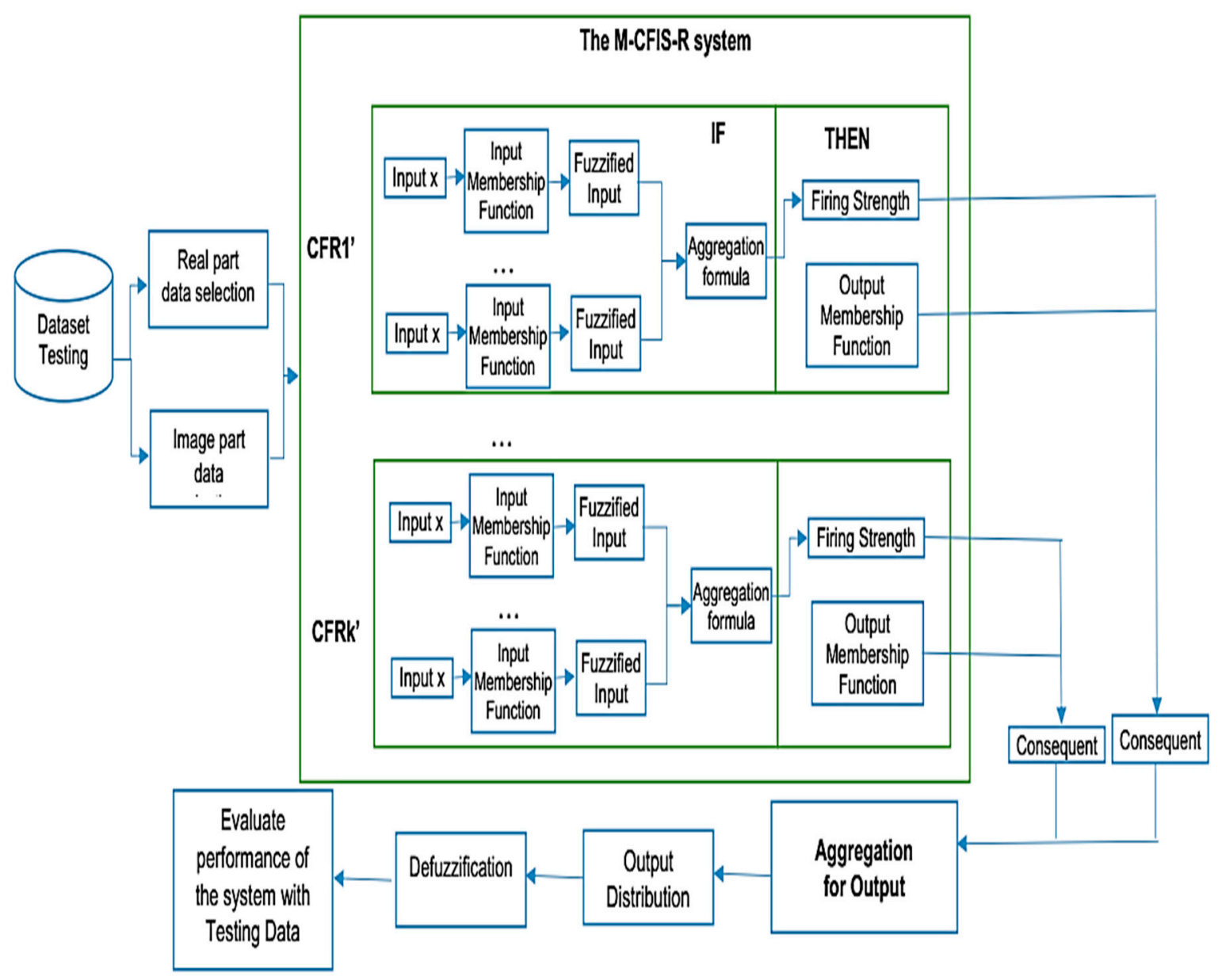

Figure 2. Testing diagram for the proposed model.

Specifically, from Testing data, we build data for the real and imaginary parts. Then, we use the reduced complex fuzzy rule base generated from the Training model to obtain the consequences. By using the Aggregation operator and Defuzzification, we obtain the output. Finally, we evaluate the performance of the outputs based on the evaluation measures (see Section 4.2.3).

\subsection{Some Notes on M-CFIS-R}

Advantages: The M-CFIS-R model combines M-CFIS, complex fuzzy measures and granular computing within the Training phase. The result is a new fuzzy rule system with better performance than M-CFIS. The novelty of this research lies on the complex fuzzy measures within granular computing. In M-CFIS [23], the Training phase was not described, so it is an advantage for this research to demonstrate the improvements in this phase. By doing so, we obtain the new M-CFIS with better complex fuzzy rule base, which results in better performance than M-CFIS [23].

Disadvantages: The new model only stopped at local optimization and did not yet obtain a global optimal solution because when evaluating the performance of a new fuzzy rule base in the Training, if it is better than that of M-CFIS, the algorithm stops. This should be enhanced further. Besides, implementation time of the proposed model is also longer than that of M-CFIS.

\section{Experiments}

\subsection{Experimental Environment}

We implemented the proposed M-CFIS-R against M-CFIS [23] in MATLAB 2014 and executed them on a PC VAIO laptop with Core i5 processor. The experimental data include two types: 
(a) Benchmark Medical UCI Machine Learning Repository Data [49]:

i. The first dataset is the Wisconsin Breast Cancer Diagnosis (WBCD) from UCI [50] with 699 examples (458 benign and 241 malignant) in nine integer inputs and one binary output (Table 1).

Table 1. Wisconsin Breast Cancer Diagnosis (WBCD) data summary.

\begin{tabular}{clc}
\hline No. & \multicolumn{1}{c}{ Feature Name } & Value Range \\
\hline 1 & Clump Thickness & $1-10$ \\
2 & Uniformity of Cell Size & $1-10$ \\
3 & Uniformity of Cell Shape & $1-10$ \\
4 & Marginal Adhesion & $1-10$ \\
5 & Single Epithelial Cell Size & $1-10$ \\
6 & Bare Nuclei & $1-10$ \\
7 & Bland Chromatin & $1-10$ \\
8 & Normal Nucleoli & $1-10$ \\
9 & Mitoses & $1-10$ \\
10 & Class & (2: benign, 4: malignant) \\
\hline
\end{tabular}

ii. The second dataset, named Diabetes Databases [51], is from the Department of Medicine of the University of Virginia School of Medicine. The data have 391 examples with two classes to test whether the patient is positive or negative for diabetes. This dataset consists of five attributes (Table 2).

Table 2. Diabetes data summary.

\begin{tabular}{clc}
\hline No & \multicolumn{1}{c}{ Feature Names } & Value Range \\
\hline 1 & Total Cholesterol & $78-443$ \\
2 & Stabilized Glucose & $48-385$ \\
3 & High Density Lipoprotein & $12-120$ \\
4 & Cholesterol/HDL Ratio & $1.5-19.3$ \\
5 & Glycosylated Hemoglobin & $2.68-16.11$ \\
6 & Class & (0: negative, 1: positive) \\
\hline
\end{tabular}

(b) Real Medical Datasets:

i. The third dataset is from Gangthep Hospital and Thai Nguyen National Hospital, Vietnam [52], including 4156 patients divided into two groups: 2954 examples of non-diseased patients and 1202 examples of diseased patients (Table 3).

Table 3. Gangthep Hospital and Thai Nguyen National Hospital data summary.

\begin{tabular}{clc}
\hline No. & \multicolumn{1}{c}{ Feature Name } & Value Range \\
\hline 1 & Age: at the exam time & $5-86$ \\
2 & Gender & $(0:$ male; 1 : female $)$ \\
3 & AST: aspartate transaminase & $11.4-659.76$ \\
4 & ALT: alanine aminotransferase & $78.52-647.7$ \\
5 & AST/ALT index & $0-8.5$ \\
6 & GGT: gamma glutamyl transferase & $0-3352.6$ \\
7 & Albumin & $0-58.2$ \\
8 & TB: Total bilirubin & $3-669.03$ \\
9 & DB: Direct bilirubin & $0-287.52$ \\
10 & DB/TB (\%) & $0-224.8$ \\
11 & Class & $(0:$ nondisease, 1 : disease $)$ \\
\hline
\end{tabular}


ii. The fourth dataset is the real dental dataset from Hanoi Medical University Hospital, Vietnam [53], in which dentists provide a properly labeled dataset that consists of 447 X-ray images with the disease of wisdom teeth deviate and $200 \mathrm{X}$-ray images without wisdom teeth deviate. The dental experts are from Hanoi Medical University and are currently working as professional dentists (Figure 3).

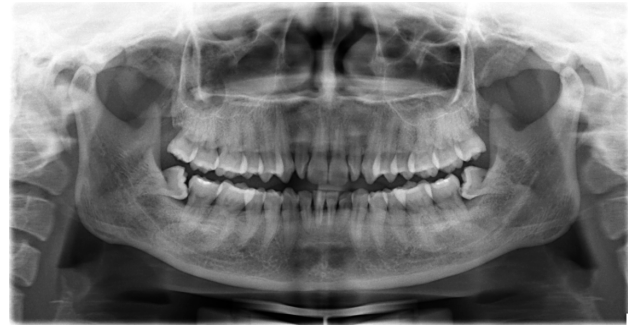

(a)

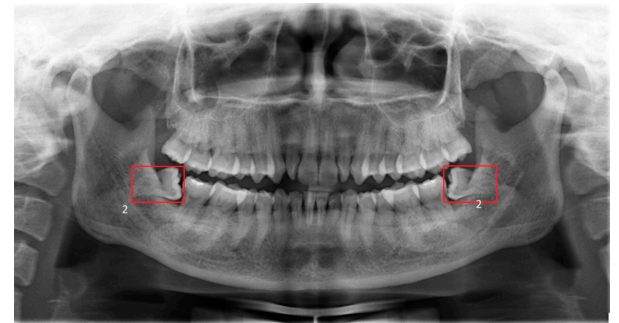

(b)

Figure 3. (a) A dental image. (b) The patient's cavity area image.

From this, we extract the following features: Gradient (GRA) [54]; Local Binary Patterns (LBP) [55]; Patch [56]; and Entropy, Edge-Value, and Intensity (EEI) [57] (Table 4). The input is an image, and the output is the label of disease or not.

Table 4. Value ranges of the dental dataset.

\begin{tabular}{ccc}
\hline ID & Features & Value Range \\
\hline 1 & LBP & $27.04-55.89$ \\
2 & EEI & $145.65-161.76$ \\
3 & GRA & $85.02-125.07$ \\
4 & Patch & $30.54 \times 10^{-3}-208.56 \times 10^{-3}$ \\
5 & Label & 0 or 1 \\
\hline
\end{tabular}

The evaluation criteria are Accuracy, Precision, and Recall, as defined in Section 4.2.3.

\subsection{Experimental Results on the Benchmark UCI Datasets}

Using 3-fold cross-validation method, the values of criteria obtained by applying M-CFIS and M-CFIS-R on the UCI datasets are visually presented in Figures 4 and 5, respectively. The average values of validity indices and time consumed are calculated separately on the training and the testing data. The number of rules is defined after applying granular computing with complex fuzzy measures. Results are taken as the average of 3-fold cross-validation. 


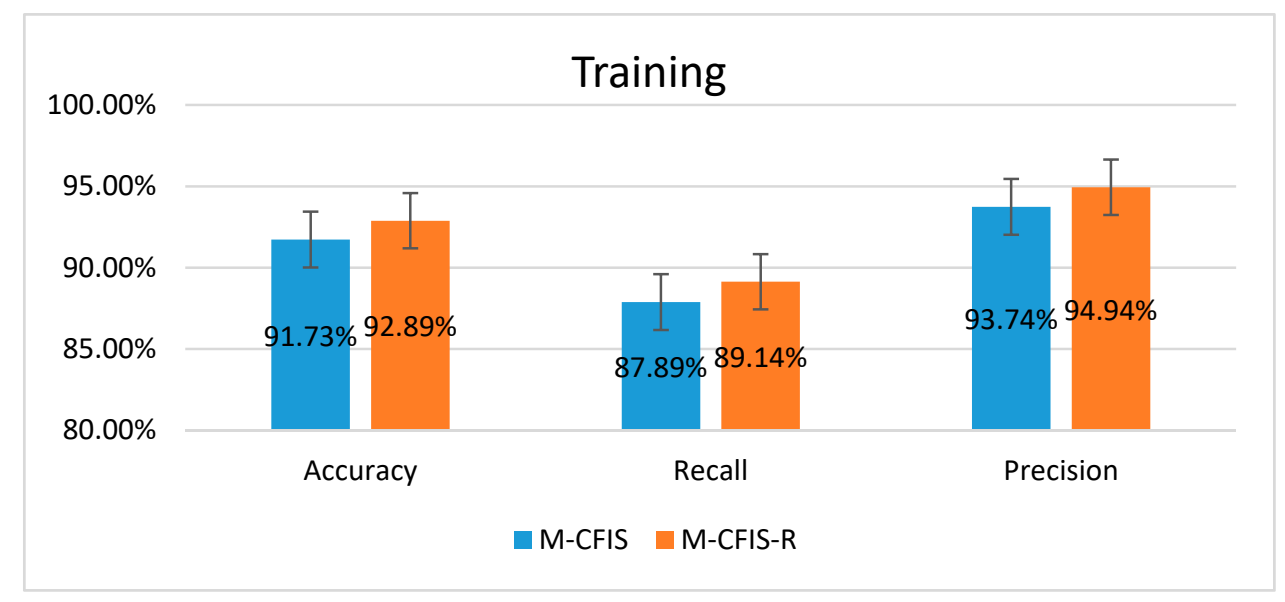

(a)

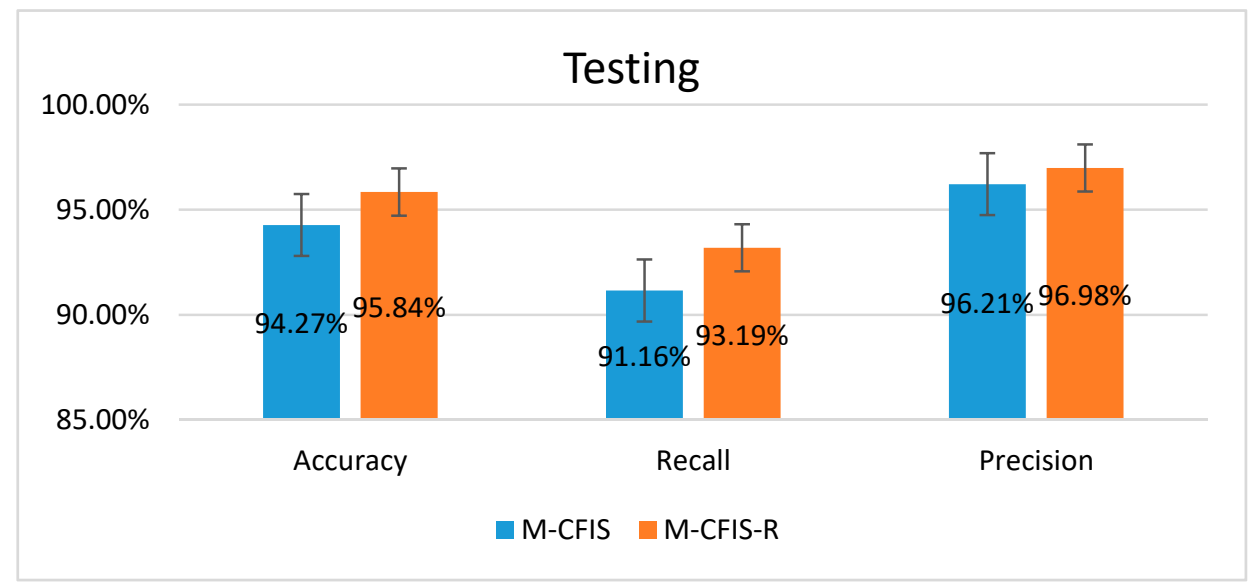

(b)

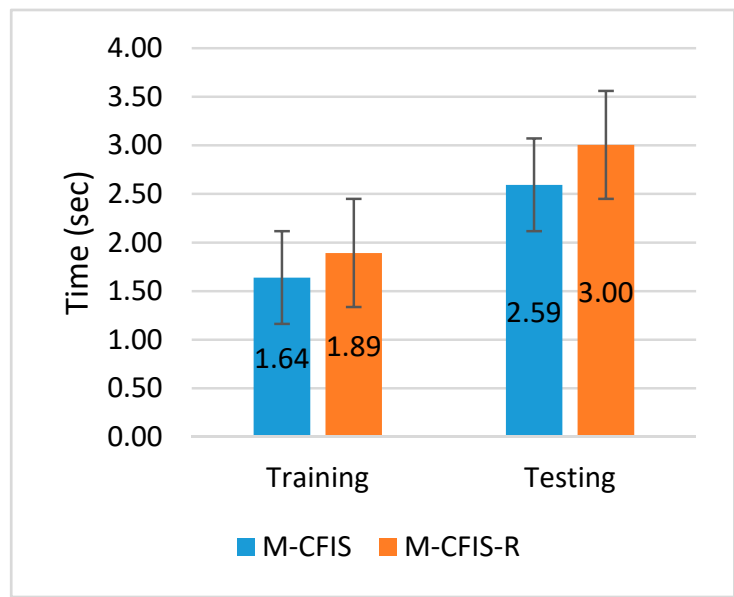

(c)

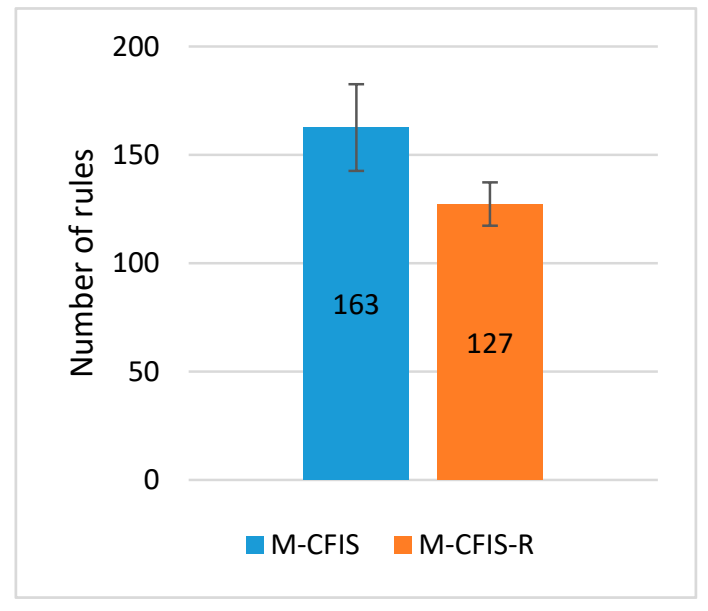

(d)

Figure 4. Performance on the WBCD dataset: (a) Accuracy, Recall, and Precision on training set; (b) Accuracy, Recall, and Precision on testing set; (c) Time consumed; (d) Number of rules. 


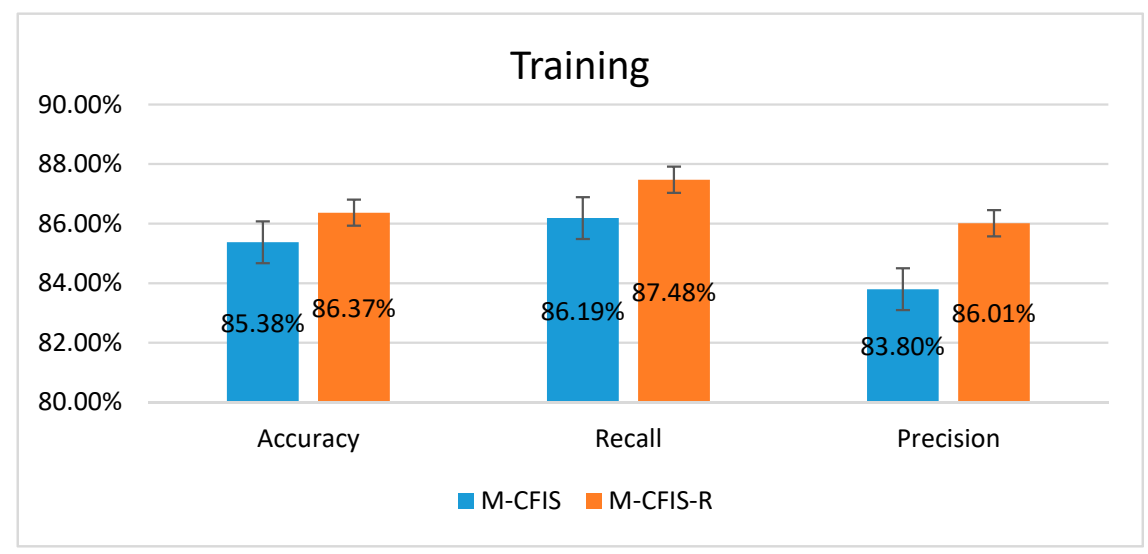

(a)

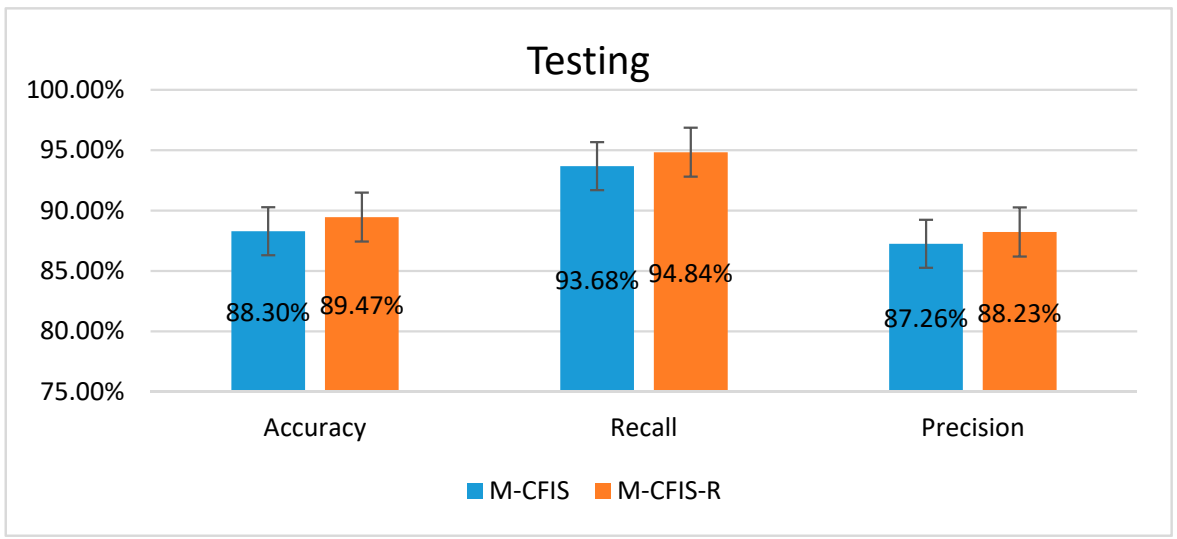

(b)

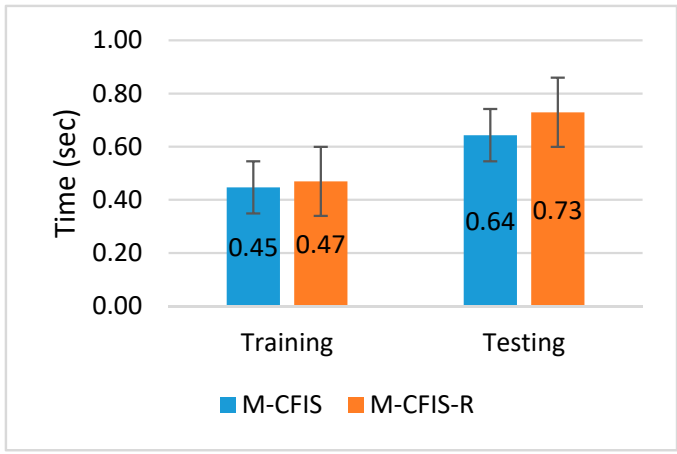

(c)

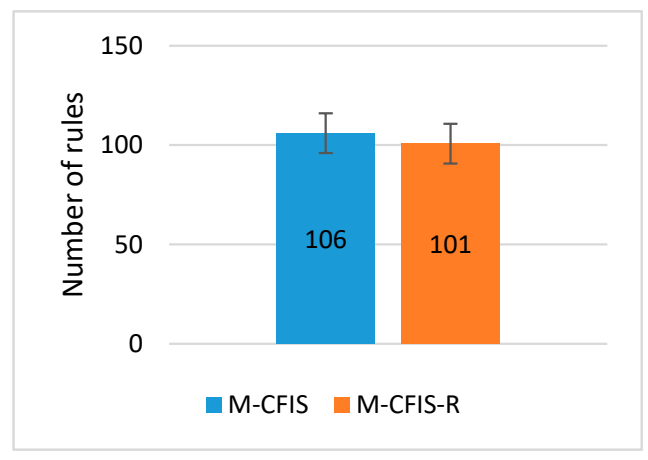

(d)

Figure 5. Performance on the Diabetes dataset: (a) Accuracy, Recall, Precision on training set; (b) Accuracy, Recall, Precision on testing set; (c) Time consumed; (d) Number of rules.

Figure 4 shows the results of applying M-CFIS and M-CFIS-R on the first dataset-WBCD. The accuracy of M-CFIS-R in the training data (Figure 4a) is higher than that of M-CFIS by $1.2 \%$ with small standard derivation (SD) (about 0.02). This value on the testing data is $1.6 \%$ higher with 0.01 of SD. Similarly, the Recall values in Figure $4 \mathrm{~b}$ of M-CFIS-R in both the training and testing data are also higher than those of CFIS with the SD being less than 0.02. The Precision values in Figure 4a,b of M-CFIS-R are a bit higher than those of M-CFIS, with very small SD (SD is even zero in the testing data).

The computation time in Figure 4c of M-CFIS-R is a bit higher than that of M-CFIS, with only $0.25 \mathrm{~s}$ on the training data and $0.41 \mathrm{~s}$ on the testing data. Thus, the computation time of these methods can be considered as equal. The average number of rules in Figure 4d of M-CFIS-R is 127 with SD of 
3.4, which is 35 rules less than the result of M-CFIS (163 rules on average with 2.06 SD). Thus, the rule base of M-CFIS-R has a lower number of rules than M-CFIS.

The performance comparison between M-CFIS and M-CFIS-R on the Diabetes dataset is presented in Figure 5. The values of validity indices (Figure 5a,b) obtained from M-CFIS-R are higher than those of M-CFIS by more than $1 \%$ and with small SD.

The running time (Figure $5 \mathrm{c}$ ) of M-CFIS-R is higher than that of M-CFIS by only $0.02 \mathrm{~s}$ on the training data and $0.086 \mathrm{~s}$ on the testing data. The standard derivations are very small as well. The computation time of M-CFIS-F is equivalent to that of M-CFIS. The average number of rules in Figure $5 \mathrm{~d}$ of M-CFIS-R is 5 rules less than that of M-CFIS, with SD of 0.94 .

\subsection{Experimental Results on the Real Datasets}

On the real datasets, the classification quality evaluation between our proposed method M-CFIS-R and M-CFIS is shown in Figures 6 and 7.

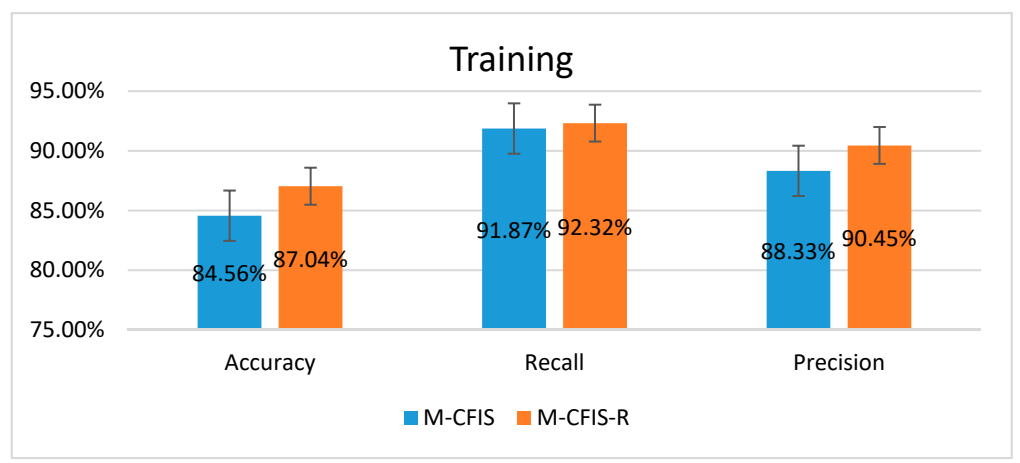

(a)

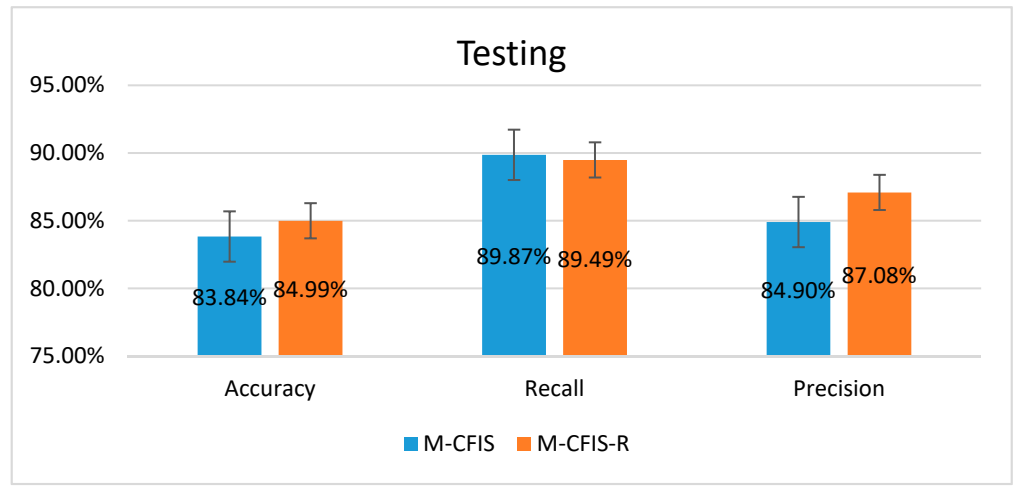

(b)

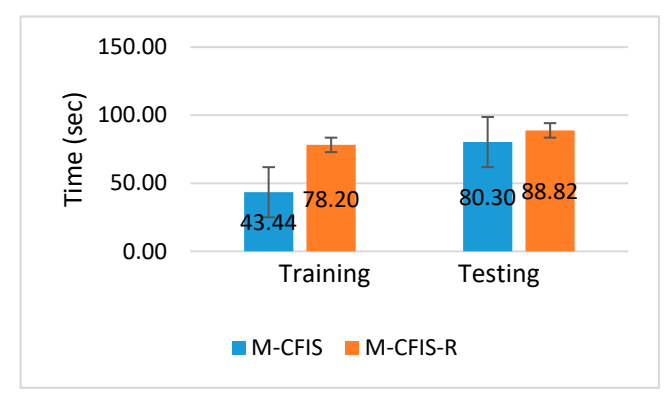

(c)

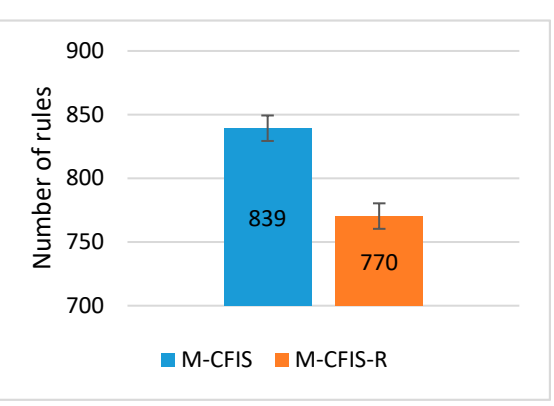

(d)

Figure 6. Performance on the Liver dataset: (a) Accuracy, Recall, Precision on training set; (b) Accuracy, Recall, Precision on testing set; (c) Time consumed; (d) Number of rules. 


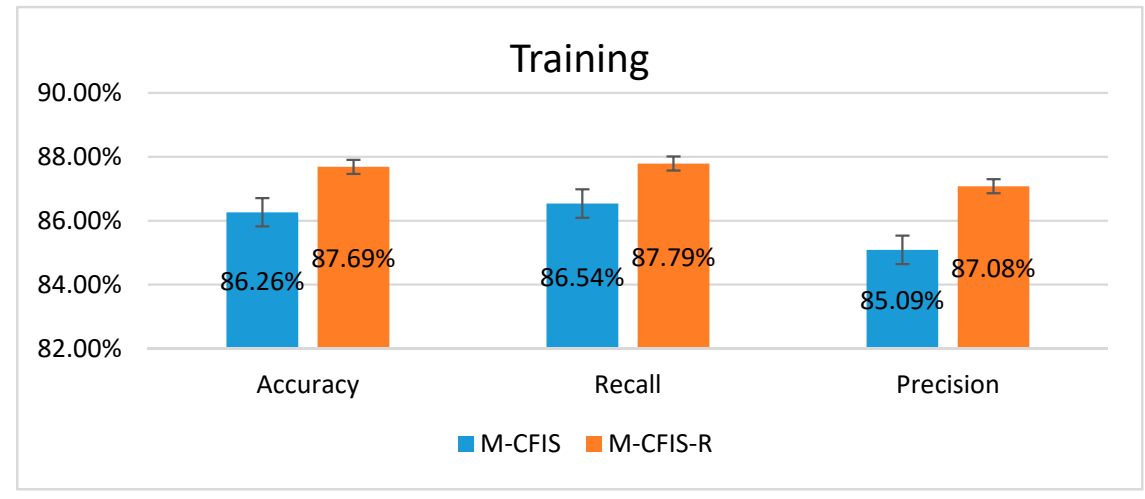

(a)

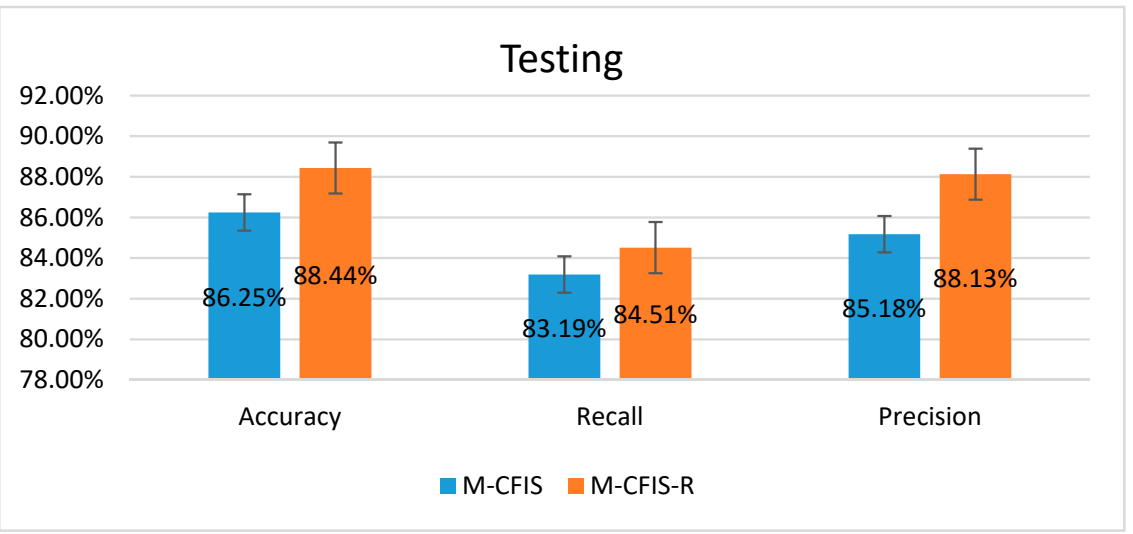

(b)

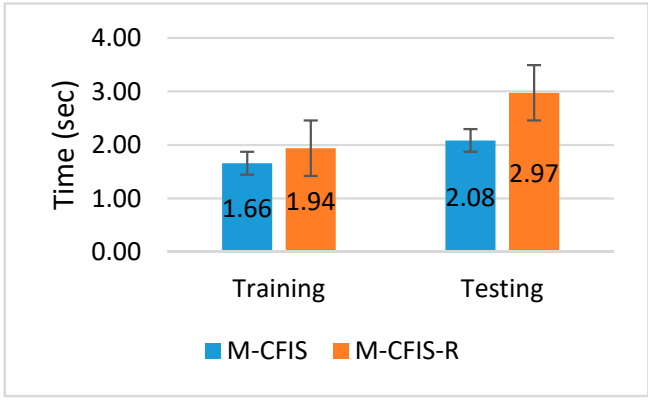

(c)

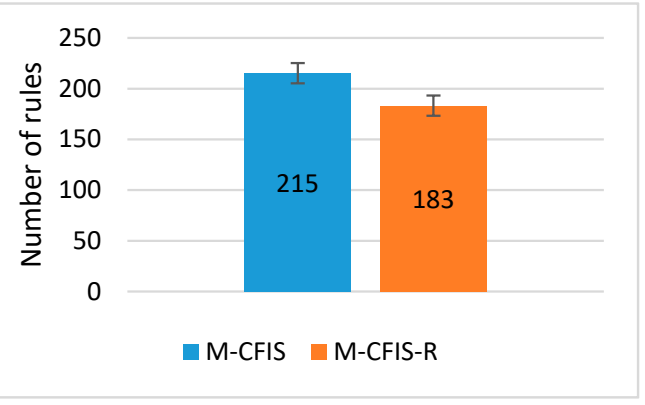

(d)

Figure 7. Performance on the Dental dataset: (a) Accuracy, Recall, Precision on training set; (b) Accuracy, Recall, Precision on testing set; (c) Time consumed; (d) Number of rules.

Figure 6 shows the performance of M-CFIS-R and M-CFIS on the Liver dataset. From Figure 6a, it is clear that the accuracy of M-CFIS-R on the training data is $2.5 \%$ higher than that of M-CFIS. Moreover, as shown in Figure $6 \mathrm{a}, \mathrm{b}$, the recall and precision values of M-CFIS-R on the training and testing data are about $2.2 \%$ higher than those of M-CFIS. Although the recall of M-CFIS-R on the testing data is $0.4 \%$ smaller than that of M-CFIS, the SD is very small (only 0.03 ). This is caused by the decreasing in number of rules, as shown in Figure 6d. On the Liver dataset, the number of rules in M-CFIS-R is 69 less than that of M-CFIS. This is the reason for M-CFIS-R being more time-consuming than M-CFIS (i.e., $34.5 \mathrm{~s}$ higher on the training data and $8.5 \mathrm{~s}$ higher on the testing data, as shown in Figure $6 \mathrm{c}$ ). The standard derivations of all these results are very low.

The performance evaluation of M-CFIS-R compared to M-CFIS on Dental dataset is presented in Figure 7. All the results in this table are mostly similar to those of three datasets mentioned above. The number of rules in M-CFIS-R is 183 rules with SD of 2.5, while the number of rules in M-CFIS 
is 215 with 3.4 SD, as shown in Figure 7d. This explains why the accuracy, precision, and recall of M-CFIS-R are higher than those of M-CFIS, as seen in Figure 7a,b.

Apart from experimental evaluation above, the qualitative comparisons between the proposed model and others are provided in Table 5.

Table 5. Theoretical comparison between the proposed work and others.

\begin{tabular}{|c|c|c|c|}
\hline Authors & Model & Brief Description & Results and Limitations \\
\hline $\begin{array}{l}\text { Selvachandran } \\
\text { et al. [23] }\end{array}$ & Mamdani CFIS & $\begin{array}{l}\text { - Extended Mamdani FIS on } \\
\text { complex fuzzy sets (Mamdani } \\
\text { CFIS) together with operations on } \\
\text { this system. } \\
\text { - Output of Mamdani CFIS is a set } \\
\text { of complex fuzzy rules used to } \\
\text { solve diagnosis problems. }\end{array}$ & $\begin{array}{l}\text { - Applying proposed model on } \\
\text { six real datasets with higher } \\
\text { accuracy than Mamdani FIS } \\
\text { and ANFIS. } \\
\text { - Limitation: There is } \\
\text { redundancy in the rule base. }\end{array}$ \\
\hline $\begin{array}{c}\text { Turabieh et al. } \\
{[58]}\end{array}$ & Dynamic ANFIS & $\begin{array}{l}\text { - An ANFIS based model to } \\
\text { predict missing values of } \\
\text { incomplete samples based on } \\
\text { complete samples. } \\
\text { - Optimized each rule in the rule } \\
\text { base using MSE. }\end{array}$ & $\begin{array}{l}\text { - The model was validated on } \\
\text { two medical datasets with } \\
\text { good results in handling } \\
\text { missing value datasets. } \\
\text { - Limitation: Unable to deal } \\
\text { with data that have phase or } \\
\text { periodic interval. }\end{array}$ \\
\hline $\begin{array}{l}\text { Ahmad et al. } \\
\text { [59] }\end{array}$ & $\begin{array}{l}\text { Multilayer } \\
\text { Mamdani FIS }\end{array}$ & $\begin{array}{l}\text { - Proposed two-stage model in } \\
\text { which Mamdani FIS is used to } \\
\text { diagnose hepatitis B. } \\
\text { - First layer determines hepatitis } \\
\text { and second layer diagnoses } \\
\text { hepatitis B. }\end{array}$ & $\begin{array}{l}\text { - Experiments were done on a } \\
\text { real dataset. The correct } \\
\text { classification rate is high. } \\
\text { - Limitation: This method is } \\
\text { restricted to medical dataset of } \\
\text { hepatitis. Does not concern } \\
\text { periodic data. }\end{array}$ \\
\hline This paper & M-CFIS-R & $\begin{array}{l}\text { - Proposed a new rule reduction } \\
\text { for M-CFIS [23] by using granular } \\
\text { computing with complex } \\
\text { similarity measures. } \\
\text { - Theoretical proofs and theorems } \\
\text { were provided. }\end{array}$ & $\begin{array}{l}\text { - Achieved high accuracy of } \\
\text { prediction in both the } \\
\text { benchmark and real datasets. } \\
\text { - Achieved the optimal number } \\
\text { of rules. } \\
\text { - Able to handle the limitations } \\
\text { of rule redundancy and } \\
\text { periodic data. } \\
\text { - Limitation: Time-consuming. }\end{array}$ \\
\hline
\end{tabular}

\section{Conclusions}

This paper proposed a new M-CFIS-R system that incorporated fuzzy similarity measures such as Complex Fuzzy Cosine Similarity Measure (CFCSM), Complex Fuzzy Dice Similarity Measure (CFDSM), and Complex Fuzzy Jaccard Similarity Measure (CFJSM) in the granular computing mechanism. The aim is to achieve a better rule base than that in the original M-CFIS system. The rule base is improved by calculating the correlations between complex fuzzy rules based on different complex fuzzy measures. The similarities of complex fuzzy rules are finally determined by granular computing according to each label of Validation data. We then evaluate performance of the new complex fuzzy rule base on the Validation data by the same inference module. If the performance is better, we end the Training and proceed to Testing; otherwise, we repeat the process of using Granular Complex Fuzzy Measure to retrain. In the Testing phase, we perform a similar procedure with M-CFIS for testing the performance of the system with the reduced complex fuzzy rule base found in the Training phase. The M-CFIS-R model combines M-CFIS, complex fuzzy measures, and granular computing within the Training phase. By doing so, we obtain the new M-CFIS with better complex fuzzy rule base, which results in better performance than M-CFIS. 
The experiments have been performed on the benchmark datasets from UCI Machine Learning Depository and real datasets from Gangthep Hospital, Thai Nguyen National Hospital, and Hanoi Medical University Hospital, Vietnam. Obviously, the results in Sections 5.2 and 5.3 clearly affirm that the proposed M-CFIS-R is better than M-CFIS in terms of accuracy, recall and precision. In general, all these indices of M-CFIS-R are higher than those of M-CFIS on average, with very low standard derivation. In most cases, the accuracy values of M-CFIS-R in the training data are smaller than those in the testing data, e.g., $92.89 \%$ vs. $95.84 \%$ on the WBCD, $86.37 \%$ vs. $89.47 \%$ on the Diabetes, and $87.69 \%$ vs. $88.44 \%$ on the Dental data. However, M-CFIS-R takes more time to identify the labels of the input samples because of using the granular computing with complex fuzzy measures. On the other hands, the rule base obtained from M-CFIS-R has better quality with a smaller number of rules than that of M-CFIS. In summary, the accuracy of M-CFIS-R is approximately $86.3-92.9 \%$ for the Training and 85-95.8\% for the Testing data. The rule reduction in M-CFIS-R compared with M-CFIS is by around 4.8-22.1\%. Lastly, M-CFIS-R is slower than M-CFIS by around 1.15 times in the Testing data on average.

However, the M-CFIS-R stops at local optimization but did not yet obtain a global optimal solution, since when evaluating the performance of a new fuzzy rule base in the Training, if it is better than that of M-CFIS, the algorithm stops. This should be enhanced further. Besides, the implementation time of the proposed model is also longer than that of M-CFIS. Different concepts of complex fuzzy measures, complex fuzzy integral, and other variants (i.e., Sugeno and Tsukamoto) of M-CFIS-R should be under investigation soon.

Author Contributions: Concept: L.H.S. and S.-Y.C.; methodology: L.H.S., L.T.H.L. and T.T.N.; software: L.T.H.L., T.M.T. and M.A.; validation: T.T.N., T.M.T. and N.L.G.; data curation: L.T.H.L. and T.M.T.; writing一original draft preparation: M.A. and L.T.H.L.; writing-review and editing: T.T.N., L.H.S., N.L.G. and S.-Y.C. All authors have read and agreed to the published version of the manuscript.

Funding: This research has been funded in part by the Graduate University of Science and Technology under grant number GUST.STS.ĐT2018-TT01. This work was supported in part by the Taiwan Building Technology Center from the Featured Areas Research Center Program within the framework of the Higher Education Sprout Project by the Ministry of Education in Taiwan.

Acknowledgments: We are grateful for the support from the staff of the Institute of Information Technology, Vietnam Academy of Science and Technology.

Conflicts of Interest: The authors declare no conflict of interest.

\section{References}

1. Zadeh, L.A. Fuzzy sets. Inf. Control 1965, 8, 338-353. [CrossRef]

2. Troussas, C.; Chrysafiadi, K.; Virvou, M. An intelligent adaptive fuzzy-based inference system for computer-assisted language learning. Expert Syst. Appl. 2019, 127, 85-96. [CrossRef]

3. Tiwari, L.; Raja, R.; Sharma, V.; Miri, R. Fuzzy Inference System for Efficient Lung Cancer Detection. In Computer Vision and Machine Intelligence in Medical Image Analysis; Springer: Singapore, 2020; pp. 33-41.

4. Sagir, A.M.; Sathasivam, S. A Novel Adaptive Neuro Fuzzy Inference System Based Classification Model for Heart Disease Prediction. Pertanika J. Sci. Technol. 2017, 25, 43-56.

5. Afriyie Mensah, R.; Xiao, J.; Das, O.; Jiang, L.; Xu, Q.; Alhassan, M.O. Application of Adaptive Neuro-Fuzzy Inference System in Flammability Parameter Prediction. Polymers 2020, 12, 122. [CrossRef]

6. Bakhshipour, A.; Zareiforoush, H.; Bagheri, I. Application of decision trees and fuzzy inference system for quality classification and modeling of black and green tea based on visual features. J. Food Meas. Charact. 2020, 1-15. [CrossRef]

7. Manogaran, G.; Varatharajan, R.; Priyan, M.K. Hybrid recommendation system for heart disease diagnosis based on multiple kernel learning with adaptive neuro-fuzzy inference system. Multimed. Tools Appl. 2018, 77, 4379-4399. [CrossRef]

8. Handoyo, S.; Kusdarwati, H. Implementation of Fuzzy Inference System for Classification of Dengue Fever on the villages in Malang. In IOP Conference Series: Materials Science and Engineering; IOP Publishing: Bristol, UK, 2019; Volume 546, p. 052026. 
9. Shastry, K.A.; Sanjay, H.A. Adaptive Neuro-Fuzzy Inference System in Agriculture. In Fuzzy Expert Systems and Applications in Agricultural Diagnosis; IGI Global: Hershey, PA, USA, 2020; pp. 130-153.

10. Abdolkarimi, E.S.; Mosavi, M.R. Wavelet-adaptive neural subtractive clustering fuzzy inference system to enhance low-cost and high-speed INS/GPS navigation system. GPS Solut. 2020, 24, 36. [CrossRef]

11. Pourjavad, E.; Shahin, A. The application of Mamdani fuzzy inference system in evaluating green supply chain management performance. Int. J. Fuzzy Syst. 2018, 20,901-912. [CrossRef]

12. Lima-Junior, F.R.; Carpinetti, L.C.R. An adaptive network-based fuzzy inference system to supply chain performance evaluation based on SCOR ${ }^{\circledR}$ metrics. Comput. Ind. Eng. 2020, 139, 106191. [CrossRef]

13. Priyadarshi, N.; Azam, F.; Sharma, A.K.; Vardia, M. An Adaptive Neuro-Fuzzy Inference System-Based Intelligent Grid-Connected Photovoltaic Power Generation. In Advances in Computational Intelligence; Springer: Singapore, 2020; pp. 3-14.

14. Adoko, A.C.; Yagiz, S. Fuzzy Inference System-Based for TBM Field Penetration Index Estimation in Rock Mass. Geotech. Geol. Eng. 2019, 37, 1533-1553. [CrossRef]

15. Ramot, D.; Milo, R.; Friedman, M.; Kandel, A. Complex fuzzy sets. IEEE Trans. Fuzzy Syst. 2002, 10, 171-186. [CrossRef]

16. Ramot, D.; Friedman, M.; Langholz, G.; Kandel, A. Complex fuzzy logic. IEEE Trans. Fuzzy Syst. 2003, 11, 450-461. [CrossRef]

17. Ngan, T.T.; Lan, L.T.H.; Ali, M.; Tamir, D.; Son, L.H.; Tuan, T.M.; Rishe, N.; Kandel, A. Logic connectives of complex fuzzy sets. Rom. J. Inf. Sci. Technol. 2018, 21, 344-358.

18. Ali, M.; Smarandache, F. Complex neutrosophic set. Neural Comput. Appl. 2017, 28, 1817-1834. [CrossRef]

19. Ali, M.; Dat, L.Q.; Smarandache, F. Interval complex neutrosophic set: Formulation and applications in decision-making. Int. J. Fuzzy Syst. 2018, 20, 986-999. [CrossRef]

20. Greenfield, S.; Chiclana, F.; Dick, S. Interval-valued complex fuzzy logic. In Proceedings of the 2016 IEEE International Conference on Fuzzy Systems (FUZZ-IEEE), Vancouver, BC, Canada, 24-29 July 2016; pp. 2014-2019.

21. Garg, H.; Rani, D. Some generalized complex intuitionistic fuzzy aggregation operators and their application to multicriteria decision-making process. Arabian J. Sci. Eng. 2019, 44, 2679-2698. [CrossRef]

22. Man, J.Y.; Chen, Z.; Dick, S. Towards inductive learning of complex fuzzy inference systems. In Proceedings of the NAFIPS 2007-2007 Annual Meeting of the North American Fuzzy Information Processing Society, San Diego, CA, USA, 24-27 June 2007; pp. 415-420.

23. Selvachandran, G.; Quek, S.G.; Lan, L.T.H.; Giang, N.L.; Ding, W.; Abdel-Basset, M.; Albuquerque, V.H.C. A New Design of Mamdani Complex Fuzzy Inference System for Multi-attribute Decision Making Problems. IEEE Trans. Fuzzy Syst. 2019. [CrossRef]

24. Tu, C.H.; Li, C. Multiple Function Approximation-A New Approach Using Complex Fuzzy Inference System. In Asian Conference on Intelligent Information and Database Systems; Springer: Cham, Switzerland, 2018; pp. 243-254.

25. Chen, Z.; Aghakhani, S.; Man, J.; Dick, S. ANCFIS: A neurofuzzy architecture employing complex fuzzy sets. IEEE Trans. Fuzzy Syst. 2010, 19, 305-322. [CrossRef]

26. Liu, Y.; Liu, F. An adaptive neuro-complex-fuzzy-inferential modeling mechanism for generating higher-order TSK models. Neurocomputing 2019, 365, 94-101. [CrossRef]

27. Yazdanbakhsh, O.; Dick, S. FANCFIS: Fast adaptive neuro-complex fuzzy inference system. Int. J. Approx. Reason. 2019, 105, 417-430. [CrossRef]

28. Alkouri, A.U.M.; Salleh, A.R. Linguistic variable, hedges and several distances on complex fuzzy sets. J. Intell. Fuzzy Syst. 2014, 26, 2527-2535. [CrossRef]

29. Hu, B.; Bi, L.; Dai, S.; Li, S. Distances of complex fuzzy sets and continuity of complex fuzzy operations. J. Intell. Fuzzy Syst. 2018, 35, 2247-2255. [CrossRef]

30. Dai, S.; Bi, L.; Hu, B. Distance measures between the interval-valued complex fuzzy sets. Mathematics 2019, 7, 549. [CrossRef]

31. Setnes, M.; Babuska, R.; Kaymak, U.; van Nauta Lemke, H.R. Similarity measures in fuzzy rule base simplification. IEEE Trans. Syst. Man Cybern. Part B Cybern. 1998, 28, 376-386. [CrossRef] [PubMed]

32. Mondal, K.; Pramanik, S.; Giri, B.C. Some similarity measures for MADM under a complex neutrosophic set environment. In Optimization Theory Based on Neutrosophic and Plithogenic Sets; Academic Press: Cambridge, MA, USA, 2020; pp. 87-116. 
33. Rani, P.; Mishra, A.R.; Rezaei, G.; Liao, H.; Mardani, A. Extended Pythagorean fuzzy TOPSIS method based on similarity measure for sustainable recycling partner selection. Int. J. Fuzzy Syst. 2020, 22, 735-747. [CrossRef]

34. Jang, L.C.; Kim, H.M. On Choquet integrals with respect to a fuzzy complex valued fuzzy measure of fuzzy complex valued functions. Int. J. Fuzzy Log. Intell. Syst. 2018, 10, 224-229. [CrossRef]

35. Jang, L.C.; Kim, H.M. Some Properties of Choquet Integrals with Respect to a Fuzzy Complex Valued Fuzzy Measure. Int. J. Fuzzy Log. Intell. Syst. 2011, 11, 113-117. [CrossRef]

36. Ma, S.; Li, S. Complex fuzzy set-valued Complex fuzzy Measures and their properties. Sci. World J. 2014. [CrossRef]

37. Ma, S.Q.; Chen, M.Q.; Zhao, Z.Q. The Complex Fuzzy Measure. In Fuzzy Information Engineering and Operations Research Management; Springer: Berlin/Heidelberg, Germany, 2014; pp. 137-145.

38. Ma, S.Q.; Li, S.G. Complex Fuzzy Set-Valued Complex Fuzzy Integral and Its Convergence Theorem. In Fuzzy Systems Operations Research and Management; Springer: Cham, Switzerland, 2016; pp. 143-155.

39. Garg, H.; Rani, D. Some results on information measures for complex intuitionistic fuzzy sets. Int. J. Intell. Syst. 2019, 34, 2319-2363. [CrossRef]

40. Ngan, R.T.; Ali, M.; Tamir, D.E.; Rishe, N.D.; Kandel, A. Representing complex intuitionistic fuzzy set by quaternion numbers and applications to decision making. Appl. Soft Comput. 2020, 87, 105961. [CrossRef]

41. Yazdanbakhsh, O.; Dick, S. Forecasting of multivariate time series via complex fuzzy logic. IEEE Trans. Syst. Man Cybern. Syst. 2017, 47, 2160-2171. [CrossRef]

42. Tu, C.H.; Li, C. Multitarget prediction-A new approach using sphere complex fuzzy sets. Eng. Appl. Artif. Intell. 2019, 79, 45-57. [CrossRef]

43. Li, C.; Tu, C.H. Complex neural fuzzy system and its application on multi-class prediction-A novel approach using complex fuzzy sets, IIM and multi-swarm learning. Appl. Soft Comput. 2019, 84, 105735. [CrossRef]

44. Singh, P.K. Granular-based decomposition of complex fuzzy context and its analysis. Prog. Artif. Intell. 2019, 8, 181-193. [CrossRef]

45. Zhang, G.; Dillon, T.S.; Cai, K.Y.; Ma, J.; Lu, J. Operation properties and $\delta$-equalities of complex fuzzy sets. Int. J. Approx. Reason. 2009, 50, 1227-1249. [CrossRef]

46. Bargiela, A.; Pedrycz, W. Granular computing. In Handbook on Computational Intelligence: Volume 1: Fuzzy Logic, Systems, Artificial Neural Networks, and Learning Systems; World Scientific publishing: Singapore, 2016; pp. 43-66.

47. Liu, H.; Cocea, M. Granular computing-based approach of rule learning for binary classification. Granul. Comput. 2019, 4, 275-283. [CrossRef]

48. Bezdek, J.C. Pattern Recognition with Ffuzzy Objective Function Algorithms; Plenum Press: New York, NY, USA, 1981.

49. The UCI Machine Learning Repository. Available online: http://archive.ics.uci.edu/ml/datasets.html (accessed on 9 April 2020).

50. Breast Cancer. Available online: http://archive.ics.uci.edu/ml/datasets/breast+cancer+wisconsin+\%28original $\%$ 29 (accessed on 9 April 2020).

51. Diabetes Databases. Available online: http://biostat.mc.vanderbilt.edu/wiki/Main/DataSets (accessed on 9 April 2020).

52. Gangthep Hospital. Available online: http://benhviengangthep.gov.vn/ (accessed on 9 April 2020).

53. Hanoi Medical University Hospital. Available online: http://benhviendaihocyhanoi.com/ (accessed on 9 April 2020).

54. Ghazali, K.H.; Mustafa, M.M.; Hussain, A.; Bandar, M.E.C.; Kuantan, G. Feature Extraction technique using SIFT keypoints descriptors. In Proceedings of the The International Conference on Electrical and Engineering and Informatics Institut Technology, Institut Teknologi Bandung, Bandung, Indonesia, 17-19 June 2007; pp. 17-19.

55. Ahonen, T.; Hadid, A.; Pietikainen, M. Face description with local binary patterns: Application to face recognition. IEEE Trans. Pattern Anal. Mach. Intell. 2006, 28, 2037-2041. [CrossRef]

56. Oad, K.K.; DeZhi, X.; Butt, P.K. A Fuzzy Rule Based Approach to Predict Risk Level of Heart Disease. Glob. J. Comput. Sci. Technol. 2014, 14, 16-22. 
57. Lai, Y.H.; Lin, P.L. Effective segmentation for dental X-ray images using texture-based fuzzy inference system. In International Conference on Advanced Concepts for Intelligent Vision Systems; Springer: Berlin/Heidelberg, Germany, 2008; pp. 936-947.

58. Turabieh, H.; Mafarja, M.; Mirjalili, S. Dynamic Adaptive Network-Based Fuzzy Inference System (D-ANFIS) for the Imputation of Missing Data for Internet of Medical Things Applications. IEEE Internet Things J. 2019, 6, 9316-9325. [CrossRef]

59. Ahmad, G.; Khan, M.A.; Abbas, S.; Athar, A.; Khan, B.S.; Aslam, M.S. Automated diagnosis of hepatitis b using multilayer mamdani fuzzy inference system. J. Healthc. Eng. 2019. [CrossRef]

(C) 2020 by the authors. Licensee MDPI, Basel, Switzerland. This article is an open access article distributed under the terms and conditions of the Creative Commons Attribution (CC BY) license (http://creativecommons.org/licenses/by/4.0/). 